\title{
Dynamical Evolution in Noncommutative Discrete Phase Space and the Derivation of Classical Kinetic Equations
}

\author{
A. Dimakis \\ Department of Mathematics, University of the Aegean \\ GR-83200 Karlovasi, Samos, Greece \\ C. Tzanakis门 \\ Department of Education, University of Crete \\ GR-74100 Rethymnon, Crete, Greece
}

\begin{abstract}
By considering a lattice model of extended phase space, and using techniques of noncommutative differential geometry, we are led to: (a) the conception of vector fields as generators of motion and transition probability distributions on the lattice; (b) the emergence of the time direction on the basis of the encoding of probabilities in the lattice structure; (c) the general prescription for the observables' evolution in analogy with classical dynamics. We show that, in the limit of a continuous description, these results lead to the time evolution of observables in terms of (the adjoint of) generalized Fokker-Planck equations having: (1) a diffusion coefficient given by the limit of the correlation matrix of the lattice coordinates with respect to the probability distribution associated with the generator of motion; (2) a drift term given by the microscopic average of the dynamical equations in the present context. These results are applied to 1D and 2D problems. Specifically, we derive: (I) The equations of diffusion, Smoluchowski and Fokker-Planck in velocity space, thus indicating the way random walk models are incorporated in the present context; (II) Kramers' equation, by further assuming that, motion is deterministic in coordinate space.
\end{abstract}

\footnotetext{
*dimakis@aegean.gr

†tzanakis@edc.uoc.gr
} 


\section{Introduction}

In the last few years there has been an increasing interest in exploring the possible relevance of noncommutative geometry (NCG) in various areas of physics. Roughly speaking, the basic idea in this context, is to try to develop an appropriate conceptual and mathematical framework in which the fundamental object is no longer a manifold (intuitively conceived as the generalization of a geometrical surface), but rather an appropriate algebra $\mathcal{A}$ (e.g. of $\mathbb{R}$ or $\mathbb{C}$ functions on it). This is (partly) motivated by the well-known result that commutative $C^{*}$-algebras correspond biuniquely to locally compact topological spaces, hence that all relevant information for the topological structure of such spaces is encoded in their algebra of functions (e.g. [1]).

From an algebraic point of view there are two possible lines of approach, depending on the commutativity or noncommutativity of the algebras considered. Though the latter has received considerable attention, especially in connection with the study of quantum groups and quantum field theory ( 2, 3, 4, 5, 6] and references therein), it is true that even if the algebra $\mathcal{A}$ is commutative, the resulting structures are quite rich, allowing for a considerable generalization of familiar geometric concepts, while keeping a rather close contact with ordinary (commutative) differential geometry. In particular, geometrical concepts and methods may be developed on both "continuous" and discrete sets, with interesting applications in such diverse fields as riemannian geometry, gauge field theory, integrable dynamical systems, stochastic calculus, symplectic mechanics and kinetic theory ([7]-[17]; for a review see [18, [19] and references there in). In this connection, a basic tool is provided by the (great variety of) differential calculi that can be defined on a commutative algebra $\mathcal{A}$ with unit 1 , generalizing the familiar (exterior) calculus of differential forms. More specifically, a differential calculus (DC) on a commutative algebra $\mathcal{A}$, is a graded associative, in general noncommutative algebra

$$
\Omega(\mathcal{A}):=\bigoplus_{r \in \mathbb{N}_{0}} \Omega^{r}(\mathcal{A})
$$

where $\Omega^{0}(\mathcal{A})=\mathcal{A}, \Omega^{r}(\mathcal{A})$ is an $\mathcal{A}$-bimodule, equipped with an exterior derivative, i.e. a linear operator

$$
\begin{gathered}
\mathrm{d}: \Omega \rightarrow \Omega, \quad \mathrm{d}^{2}=0, \quad \mathrm{~d}(1)=0 \\
\mathrm{~d}\left(\omega \omega^{\prime}\right)=(\mathrm{d} \omega) \omega^{\prime}+(-1)^{r} \omega \mathrm{d} \omega^{\prime}, \quad \omega \in \Omega^{r}(\mathcal{A})
\end{gathered}
$$

Elements of $\Omega^{r}(\mathcal{A})$ are called (differential) $r$-forms and we assume that as an $\mathcal{A}$-bimodule, $\Omega^{r}(\mathcal{A})$ is generated by $\mathrm{d}\left(\Omega^{r-1}(\mathcal{A})\right)$.

A key concept here, allowing for interesting conceptual insights, is the commutative and associative product of 1-forms defined byt

$$
\omega \bullet \mathrm{d} f:=[\omega, f]:=\omega f-f \omega, \quad f \in \mathcal{A}, \omega \in \Omega^{1}
$$

\footnotetext{
${ }^{1}$ From now on we write $\Omega^{r}$ instead of $\Omega^{r}(\mathcal{A})$ and the symbol ":=" indicates a defining relation for the object that lies on the side of ":".
} 
and extended bilinearly in $\mathcal{A}$, so that

$$
(f \omega g) \bullet\left(f^{\prime} \omega^{\prime} g^{\prime}\right)=f f^{\prime}\left(\omega \bullet \omega^{\prime}\right) g g^{\prime}, \quad f, f^{\prime}, g, g^{\prime} \in \mathcal{A}, \omega, \omega^{\prime} \in \Omega^{1}
$$

(for more details, see [20], $\S \S 2,3$ ). For the existence of this $\bullet$ product, commutativity of $\mathcal{A}$ is essential.

In the usual DC, eq(1.3) is zero so that $\bullet$ is trivial. In fact, by (1.3), the Leibniz rule (1.2) can be written in $\Omega^{1}$ as

$$
\mathrm{d}(f g)=f \mathrm{~d} g+g \mathrm{~d} f+\mathrm{d} f \bullet \mathrm{d} g
$$

Thus (1.5) stresses the nature of noncommutative DC as a deformation of the usual DC. On the other hand, it is formally identical with the generalized Itô differentiation rule in Stochastic Calculus (StC), $f, g$ being semimartingales and the 3rd term in (1.5) is the socalled bracket of the processes, related to their quadratic variation ([21, appendix eq(4)). In fact, it can be shown that this is not merely a formal analogy; more precisely, a noncommutative product can be easily defined for semimartingales and their differentials, so that the stochastic differentiation rule becomes identical to (1.5) ([9] §3). In this way one can obtain results of StC by employing techniques of NCG (e.g. see [22] $\S 3$ for the Ornstein-Uhlenbeck process)

Motivated by the above remarks, one may develop differential geometry on the basis of a minimal deformation of the usual DC, namely, a calculus in which

$$
\mathrm{d} f \bullet \mathrm{d} g \bullet \mathrm{d} h=[[\mathrm{d} h, g], f]=0
$$

and explore its relation to StC. Further motivation for this, stems from the following remarks:

From a physical point of view, StC is a mathematical formalism on the basis of which a precise meaning can be given to stochastic dynamical models of physical systems whose time evolution cannot be given in terms of deterministic flows in their phase space (e.g. brownian motion). These are systems for which the concept of a trajectory in phase space is not defined. Mathematically speaking, their evolution cannot be described by semigroups of Perron-Frobenius operators, since their states often evolve under a 2nd order differential operator (differential operators can generate Perron-Frobenius semigroups only if they are of the first order, see e.g. [23], ch.7). The appearance of such operators is a basic feature of StC (via Itô's formula). In this way, by appropriate generalizations of basic geometrical concepts in the context of StC, a general mathematical framework results for the description of such nondeterministic systems $([2]])$. On the other hand, 2nd order evolution equations are at the heart of kinetic theory, for describing time-irreversible evolution towards equilibrium; they are obtained by employing some approximation scheme to the time-reversible microscopic dynamics.

Taking account of the above discussion and starting with (1.6), we have formulated basic concepts of differential geometry, tensor calculus and symplectic mechanics in a noncommutative context and we have shown that hamiltonian equations for observables are (adjoint to) generalized Fokker-Planck (FP) equations like those encountered in Itô's StC and the kinetic theory of open systems ([13], [15]).

These are suggestive mathematical developments for the relevance of NCG to StC and kinetic theory. However, from a conceptual point of view, the physical meaning of noncommutativity has to be made clearer, particularly in connection with the time evolution of 
physical systems. On this issue, a basic intuitive idea is that noncommutativity is due to the fact that differentials (i.e. 1-forms) have a "size", so that different results are obtained when functions are evaluated at the left end of the 1-form (its multiplication by functions from the left) and at the right end of it (multiplication from the right) - cf. [13], §6. On the other hand, 2nd order FP equations result in the context of kinetic theory (based either on microscopic dynamics or stochastic models), by assuming that, although individual microscopic interactions happen on a time-scale very short compared to the (macroscopic) relaxation time of the system as a whole, yet this microscopic time-scale is long enough to allow for many changes in the microscopic configurations of the system (see e.g. 224 §§II.1, II.4, 25] ch.IX.4). Therefore, although eventually one passes to the limit of a continuous description in terms of macroscopic kinetic equations, the fine structure of the system on the "infinitesimal" microscopic time-scale has already been taken into account. This is a methodology widely used in statistical mechanics and kinetic theory; to start from a discretized picture of the system and subsequently pass to a continuous macroscopic description in some appropriate limit (e.g. thermodynamic limit, appropriate scaling of the physical quantities, [26] ch.3.3, [27], §I, [28], [29] ch.III).

On the basis of this, we may try to develop a discrete analogue of the formalism in 13], 15, aiming at the following: (a) To throw more light into the physical meaning of noncommutativity of the DC; (b) to understand better the similarity of the DC to StC and (c) to provide a method for deriving irreversible evolution equations, which describe nondeterministic motions and which can be given a probabilistic interpretation, as exact rather than approximate results, much in the spirit of [13], $\S 1$, and in contrast with the general methodology underlying statistical mechanics (cf. the discussion in [13] §1). In In this connection, the techniques developed in [11], [17] for NCG on discrete sets will be used, since, on the one hand they allow for a clear geometric representation of noncommutative DC and on the other hand, they are conceptually closer to those used in the study of specific models in stochastic dynamics or statistical mechanics. 3

In view of the above, in the present paper we will show how simple model kinetic equations can be derived in the context of DC on a discrete set, having a three-fold aim:

(i) From the conceptual point of view, to clarify the relevance of noncommutative DC to kinetic theory and more generally, to the description of physical systems in terms of irreversible evolution equations;

(ii) since in kinetic theory and StC, probabilistic concepts are central, to explain how such concepts could naturally arise in the context of DC applied to systems conventionally studied by kinetic theory and stochastic dynamics;

(iii) in analogy with classical dynamics, to formulate a general prescription for what dynamical evolution means in a formalism based on NCG.

More specifically, the paper is organized as follows:

The basic ideas in this section and the above general aims of the paper, are already appar-

\footnotetext{
${ }^{2}$ Notice that, implicit to the derivation of kinetic equations by approximating microscopic dynamics, is the assumption that the higher the approximation, the better are the resulting equations. However, this usually leads to differential equations of order higher than the second, in direct conflict with the fact that at least for linear autonomous equations, it is known that only differential operators of order at most 2 can generate semigroups of solutions admitting a probabilistic interpretation, [30].

${ }^{3}$ Though formalisms based on the "continuum", more often than not can be manipulated mathematically more easily than formalisms based on discrete methods, the latter often provide deeper conceptual insights.
} 
ent in the 1D-model considered in section 2. This fact suggests the line of approach in the subsequent sections. The basic ideas are introduced systematically in section 3. Specifically, (a) the extended phase space as a discrete set, in particular a hypercubic oriented lattice,

(b) vector fields as generators of motion and probability distributions, (c) the emergence of the time direction on the basis of the encoding of probabilities in the lattice structure of the extended phase space, $(\mathrm{d})$ the general prescription of the evolution equation for observables, in direct analogy with conventional dynamics. In section 4 we apply the previous ideas and results in 1D-problems, thus getting the incorporation of random walk models in our context and accordingly showing how the diffusion, Smoluchowski and FP equation in velocity space, result in the continuous limit. In section 5 the approach of section 4 is generalized in $N$ dimensions, giving in the continuous limit a generalized FP equation in which, (1) the diffusion matrix is nonnegative-definite and is the limit of the correlation matrix of the lattice, with respect to the probability distribution associated with the vector field which is the generator of motion; (2) the drift terms are specified by the dynamics of the particular problem under investigation. Some comments on the form and properties of the transformations in phase space, are given in section 6 . In section 7 the results of section 5 are applied to the 2D-case. As an example, Kramers' equation is derived by assuming Newtonian equations of motion with friction, and requiring that in the continuous limit, motion in coordinate space is deterministic (i.e. trajectories exist there). In section 8 the nature and mathematical properties of the limiting procedure used in this paper and of other such procedures, are examined, showing that only the limit used here is well defined for all possible (1st order) differential structures on the lattice. Moreover, if the conceptual framework of $\S 3$ is employed, then we show that the only differential operators which can be generators of evolution of observables are at most of order 2. Finally, in section 9 we summarize our results and discuss the main ideas involved, as well as possible further elaborations in the present context.

\section{A One-Dimensional Model: Motivation For Further Developments}

As already mentioned in $\S 1$, there is a formal similarity of the Leibniz rule in noncommutative DC, eq(1.5), and stochastic differentiation. Actually, in [9], it was shown that Itô's stochastic differentiation can be made an exterior derivative in noncommutative DC. In this section we explore further this issue by means of a simple 1D discrete model, following the rationale of $\S 1$. Specifically, we consider a point moving in one dimension $x$, so that motion can be described in $\mathbb{R}^{2}$ with local coordinates $(t, x)$, equipped with a DC having the following commutation relations ( $t$ is the time; for the choice of the signs see $\S 3.4$ )

$$
\mathrm{d} t \bullet \mathrm{d} t=-b \mathrm{~d} t, \quad \mathrm{~d} x \bullet \mathrm{d} x=-h \mathrm{~d} t, \quad \mathrm{~d} t \bullet \mathrm{d} x=-b \mathrm{~d} x, \quad h, b>0
$$

and we assume that $\{\mathrm{d} t, \mathrm{~d} x\}$ form a basis of 1-forms. Associativity of $\bullet$ implies that

$$
\mathrm{d} x \bullet \mathrm{d} x \bullet \mathrm{d} x=a^{2} \mathrm{~d} x, \quad a^{2}:=h b
$$

so that in the limit $a, b \rightarrow 0, a^{2} / b \rightarrow h=$ constant, we recover in one dimension, the DC of [9] $\S 5,13$ \4, in close analogy with the Itô StC and with commutation relations

$$
\mathrm{d} t \bullet \mathrm{d} t=\mathrm{d} t \bullet \mathrm{d} x=0, \quad \mathrm{~d} x \bullet \mathrm{d} x=-h \mathrm{~d} t
$$


It is a special case of the DC defined by (1.6). With the transformation

$$
u=\frac{1}{2}\left(\frac{x}{a}-\frac{t}{b}\right), v=-\frac{1}{2}\left(\frac{x}{a}+\frac{t}{b}\right) \Leftrightarrow t=-b(u+v), x=a(u-v)
$$

(2.1) becomes

$$
\mathrm{d} u \bullet \mathrm{d} u=\mathrm{d} u, \quad \mathrm{~d} u \bullet \mathrm{d} v=0, \quad \mathrm{~d} v \bullet \mathrm{d} v=\mathrm{d} v
$$

which is the DC on an oriented square lattice. Hence, from (1.3) we easily get

$$
\mathrm{d} f=(f(u+1, v)-f(u, v)) \mathrm{d} u+(f(u, v+1)-f(u, v)) \mathrm{d} v
$$

so that a direct calculation using (2.6) gives

$$
\mathrm{d} f(t, x)=\bar{\partial}_{x} f(t-b, x) \mathrm{d} x+\left(\partial_{-t} f(t, x)-\frac{h}{2} \Delta_{x} f(t-b, x)\right) \mathrm{d} t
$$

where

$$
\begin{gathered}
\left(\bar{\partial}_{x} f\right)(t, x):=\frac{f(t, x+a)-f(t, x-a)}{2 a}, \\
\left(\partial_{-t} f\right)(t, x):=\frac{f(t, x)-f(t-b, x)}{b} \\
\left(\Delta_{x} f\right)(t, x):=\frac{f(t, x+a)+f(t, x-a)-2 f(t, x)}{a^{2}}
\end{gathered}
$$

In the limit $a, b \rightarrow 0, a^{2} / b \rightarrow h=$ constant, (2.7) becomes

$$
\mathrm{d} f(t, x)=\partial_{x} f(t, x) \mathrm{d} x+\left(\partial_{t} f(t, x)-\frac{h}{2} \partial_{x}^{2} f(t, x)\right) \mathrm{d} t
$$

recovering in 1D the results of previous works ([13] eq(4.13), [9] eq(5.3), [22] eq(5.1)) and essentially Itô's formula for the differential of a function of a Wiener process with variance $h$.

In order to explore this similarity further, we consider the (free) motion of a point in this context, by introducing the concept of a differentiable motion in analogy with the ordinary DC. To this end, let $\left(\mathbb{R}, s, \mathrm{~d}^{\prime}\right)$ be a discrete $\mathrm{DC}$ on $\mathbb{R}$ ( $s$ being a coordinate function on $\mathbb{R}$ parameterizing this motion), defined by

$$
\mathrm{d}^{\prime} s \bullet \mathrm{d}^{\prime} s=-b \mathrm{~d}^{\prime} s
$$

Then, we define a differentiable motion to be a mapping

$$
\gamma: \mathbb{R} \rightarrow \mathbb{R}^{2}, \quad \gamma(s)=(t(s), x(s))
$$


inducing a "pull-back" homomorphism $\gamma_{*}$ on $\Omega^{1}\left(\mathbb{R}^{2}, \mathrm{~d}\right)$ to $\Omega^{1}\left(\mathbb{R}, \mathrm{d}^{\prime}\right)$ with

$$
\gamma_{*}(x)=x(s), \quad \gamma_{*}(t)=t(s), \quad \gamma_{*} \circ \mathrm{d}=\mathrm{d}^{\prime} \circ \gamma_{*}
$$

$\mathrm{Eq}(2.13)$ implies

$$
\gamma_{*}(\mathrm{~d} x)=\frac{x(s)-x(s-b)}{b} \mathrm{~d}^{\prime} s=: \dot{x}(s) \mathrm{d}^{\prime} s, \quad \gamma_{*}(\mathrm{~d} t)=\frac{t(s)-t(s-b)}{b} \mathrm{~d}^{\prime} s=: \dot{t}(s) \mathrm{d}^{\prime} s
$$

Applying $\gamma_{*}$ to the commutation relations (2.1), (2.2), we get the following consistency relations

$$
b \dot{x}^{2}(s)=h \dot{t}(s), \quad b \dot{x}(s) \dot{t}(s)=b \dot{x}(s), \quad b \dot{t}^{2}(s)=b \dot{t}(s)
$$

Therefore, with $\dot{x}(s) \neq 0, \dot{t}(s) \neq 0$ to avoid trivial cases, we get $\dot{t}=1, \dot{x}= \pm a / b$, hence

$$
\frac{x(s)-x(s-b)}{b}= \pm \frac{a}{b}
$$

The following remarks can be made here:

(i) Motion consists of jumps $\pm a$ in discrete time intervals $b$, i.e (2.17) gives the discrete velocity of the point. In the continuous limit which leads to Itô's StC, (2.17) becomes infinite, strongly suggesting the picture of brownian motion as a random walk with equal left and right probabilities of jumps $\pm a$ at time intervals $b$ and diffusion constant $a^{2} / b$ (see e.g. 24] eq(17), 31], eq(7)).

(ii) The DC defined by (2.1) is up to a coordinate transformation the DC on an oriented square lattice (cf. (2.4)).

If the model is to be taken seriously, then these remarks raise the following issues:

- How can probabilistic concepts be introduced, given that no such concepts appeared above? In other words, is there some deeper reason for the formal similarity of the model with random walk, which may lead to the introduction of probabilities at a fundamental level?

- The above model corresponds to $1 D$-free motion. How can it be generalized to higher dimensions and/or other models? That is, what is the general prescription of time evolution in the context of noncommutative DC?

These questions correspond to the general aims of the paper, expressed in $\S 1$ and are discussed in the next section.

\section{Basic Ideas And General Formalism}

In $\S 1$ we noticed that in statistical mechanics and kinetic theory, one often starts from a discrete picture of the system under consideration, either for methodological reasons, or

\footnotetext{
${ }^{4}$ More generally, a mapping $\phi:\left(\Omega^{\prime}(\mathcal{A}), \mathrm{d}^{\prime}\right) \rightarrow(\Omega(\mathcal{A}), \mathrm{d})$ between two DC, is called differentiable, if it is a homomorphism of $\Omega^{\prime}(\mathcal{A})$ to $\Omega(\mathcal{A})$ and $\phi \circ \mathrm{d}^{\prime}=\mathrm{d} \circ \phi($ see [12], $\S 4)$.
} 
because at the microscopic level these systems are in some fundamental sense discrete (e.g. composed by a number of particles). This idea also appears in other areas, like lattice field theory (e.g. [32], ch.8), or certain approaches to quantum gravity (e.g. [33, 34, see also [35], [36] for recent reviews, and references in [12] \$1). Therefore, motivated by the discussion in $\S \S 1$ and 2 , we start with a discrete (finite or denumerable) set $\mathcal{M}$, which we call the "phase space" and eventually pass to the special case of an oriented hypercubic lattice $\mathbb{Z}^{N+1}$. We will use the concepts and techniques developed in [11, 12, 17, a summary of which is given below. At this point we stress that, as explained in $\S 3.1$, starting with a discrete picture has far-reaching consequences, since any differential structure on $\mathcal{M}$ is necessarily noncommutative, i.e. discretization implies noncommutativity of the DC on $\mathcal{M}$. Thus, the need for, or necessity of, a discrete description of physical objects, can be seen as a basic motivation (or explanation!) for introducing noncommutative differential structures in the study of physical systems.

\subsection{Differential calculi on a discrete set: A summary}

Let $\mathcal{A}$ be the algebra of $\mathbb{C}$-valued functions on $\mathcal{M}$, with the usual algebraic operations. Then,

$$
\begin{gathered}
f=\sum_{i \in \mathcal{M}} f(i) e_{i}=: \sum_{i \in \mathcal{M}} f_{i} e_{i}, \quad \forall f \in \mathcal{A} \\
e_{i} e_{j}=\delta_{i j} e_{i}, \quad e_{i}(j):=\delta_{i j}, \quad e_{i} \in \mathcal{A} \\
\sum_{i} e_{i}=1
\end{gathered}
$$

We introduce a $\mathrm{DC}$ on $\mathcal{A}$ as in $\S 1$. By defining

$$
e_{i j}:=\left\{\begin{array}{cc}
e_{i} \mathrm{~d} e_{j} & i \neq j \\
0 & i=j
\end{array}\right.
$$

so that

$$
f e_{i j}=f_{i} e_{i j}, \quad e_{i j} f=f_{j} e_{i j}
$$

we get $e_{i} \mathrm{~d} e_{i}=-\sum_{j \neq i} e_{i j}$ and consequently

$$
\mathrm{d} e_{i}=\sum_{j}\left(e_{j i}-e_{i j}\right)
$$

On the basis of this we can prove that $\left\{e_{i}\right\}$ is linearly independent and a basis of $\Omega^{1}$ taken as a vector space over $\mathbb{C}$. In particular

$$
\mathrm{d} f=\sum_{i, j}\left(f_{j}-f_{i}\right) e_{i j}, \quad \mathrm{~d} f \bullet e_{i j}=\left(f_{j}-f_{i}\right) e_{i j}, \quad e_{i j} \bullet e_{k l}=\delta_{i k} \delta_{j l} e_{i j}, \quad \forall f \in \mathcal{A}
$$

It can be proved $([1] \S \mathrm{II})$ that the $e_{i j}$ induce a basis on $\Omega^{r}, \quad r \geq 2$ over $\mathbb{C}$ via

$$
\left\{e_{i_{1} \cdots i_{r}} \mid e_{i_{1} \cdots i_{r}}:=e_{i_{1} i_{2}} e_{i_{2} i_{3}} \cdots e_{i_{r-1} i_{r}}\right\}
$$


Therefore, there is a simple way to obtain any particular DC on $\mathcal{A}$ simply by imposing relations among the $e_{i j}$, which turn to be equivalent to putting some of the $e_{i j}$ equal to 0 . This implies additional form relations for $r \geq 2$. Actually, the DC defined by (3.3), (3.7) is the largest one, called the universal DC on $\mathcal{A}$. At this point it is conceptually suggestive to notice that $e_{i j}$ with $i \neq j$ may be represented as an arrow from $i$ to $j, i \longmapsto j$. Then, universality means that all pairs of points of $\mathcal{M}$ are connected by two antiparralel arrows and any $\mathrm{DC}$ on $\mathcal{A}$ is obtained by simply discarding some arrows from this complete di-graph (viz. directed graph). In this representation, (3.5) gives

$$
\mathrm{d} e_{i}=(\text { sum of incoming arrows at } i)-(\text { sum of outgoing arrows at } i)
$$

and similar interpretations hold for (3.7). Moreover, (3.4) shows that left (right) multiplication of 1 -forms $e_{i j}$ by functions, implies that functions are evaluated at the starting (end) point of the corresponding arrow. This proves that any nontrivial DC on a discrete set $\mathcal{M}$ is necessarily noncommutative, since at least one $e_{i j}$ is nonzero so that $\mathrm{d} f \bullet e_{i j}$ is not identically zero. Actually, this quantity is (proportional to) the change of $f$ along the arrow $i \longmapsto j$. These remarks are important for what follows in this section.

\subsection{Vector fields on a discrete set $\mathcal{M}$}

Vector fields $X$ on $\mathcal{A}$ are elements of the $\mathcal{A}$-bimodule $\mathcal{X}(\mathcal{A})$ dual to $\Omega^{1}$, defined by

$$
\langle f \omega, g X \cdot h\rangle:=f g\langle\omega h, X\rangle, \quad f, g, h \in \mathcal{A}, \omega \in \Omega^{1}
$$

where $\langle$,$\rangle denotes duality contraction with \Omega^{1}$ considered as a left $\mathcal{A}$-module.

By (3.8), vector fields act as operators on $\mathcal{A}$

$$
X(f):=\langle\mathrm{d} f, X\rangle
$$

hence by (3.8) and the Leibniz rule

$$
(f X)(g)=f X(g), \quad(X \cdot f)(g)=X(f g)-g X(f)
$$

We define elements $\left\{\partial_{i j}\right\} \in \mathcal{X}(\mathcal{A})$, "dual" to $\left\{e_{i j}\right\}$, by

$$
\left\langle e_{i j}, \partial_{k l}\right\rangle=\delta_{i k} \delta_{j l} e_{i}
$$

(putting $\partial_{k k}=0$ ). A direct calculation, using (3.11), (3.4), gives

$$
e_{k} \partial_{i j}=\delta_{i k} \partial_{i j}, \quad \partial_{i j} \cdot e_{k}=\delta_{j k} \partial_{i j}
$$

so that

$$
\partial_{i j} \cdot f=f(j) \partial_{i j}, \quad f \partial_{i j}=f(i) \partial_{i j}, \quad \partial_{i j}(f)=(f(j)-f(i)) e_{i}
$$

Hence for any $X \in \mathcal{X}$

$$
X=\sum_{i, j} X^{i j} \partial_{i j}, \quad X(f)=\sum_{i, j}\left(f_{j}-f_{i}\right) X^{i j} e_{i}, \quad X^{i j} \in \mathbb{C}
$$


and we may write $X$ as an ordinary difference operator $X=\sum_{i, j} X^{i j} \partial_{i j}=: \sum_{\alpha} X^{\alpha} \partial_{\alpha}$, where $\alpha$ is an index for the arrows of the di-graph. The following proposition plays an important role in this paper and its proof is straightforward:

Proposition 3.1 Let I be the identity mapping on $\mathcal{A}$. Then $\phi: \mathcal{A} \rightarrow \mathcal{A}$ is an endomorphism of $\mathcal{A}$, if and only if

$$
X:=\phi-I
$$

satisfies

$$
X(f g)=g X(f)+f X(g)+X(f) X(g)
$$

We remark here that $X$ in general is not a vector field on $\mathcal{M}$. Nevertheless eq(3.13) readily implies that $\partial_{i j}$ (equivalently, $\partial_{\alpha}$ ), satisfy (3.16). Using this, we easily prove:

Proposition 3.2 A vector field $X \in \mathcal{X}(\mathcal{A})$ generates an endomorphism $\phi$ via (3.15), if and only if

$$
X=\sum_{\alpha} X^{\alpha} \partial_{\alpha}, \quad \text { with } \quad X^{\alpha} X^{\beta}-\delta^{\alpha \beta} X^{\alpha}=0
$$

$\mathrm{Eq}(3.17)$ simply means that at each point of $\mathcal{M}$, at most one of the $X^{\alpha}$ is nonzero and equal to 1 . By (3.16), (3.17), $X$ is the generator of a discrete semigroup of endomorphisms of $\mathcal{A}$, $\left\{\phi^{n}=(I+X)^{n}, n \in \mathbb{N}\right\}$.

As it is shown in appendix A.1, $\phi$ is an automorphism, if and only if it induces a 1-1 and onto mapping $\Phi: \mathcal{M} \rightarrow \mathcal{M}$. In this case, $\phi$ is essentially the Koopman operator associated with $\Phi$ and maps the basis $\left\{e_{i} \mid i \in \mathcal{M}\right\}$ onto itself (see e.g. [23] §3.3). Therefore, the orbits of the semigroup generated by $X$ in (3.17) are determined by the trajectories of points of $\mathcal{M}$ under $\Phi$ (i.e. the flow defined by $\Phi)$, which may be thought as lying along arrows of the di-graph corresponding to the $\mathrm{DC}$ on $\mathcal{M}$ (notice that by the remark above, at each point of $\mathcal{M}$ at most one term in $(3.17 \mathrm{i})$ is nonzero, and by (3.13) each $\partial_{i j}$ gives the change of functions along the arrow $i \longmapsto j$ ). Thus, vector fields generating automorphisms of $\mathcal{A}$, define flows on $\mathcal{M}$ along arrows of the di-graph that map points of $\mathcal{M}$ to points of $\mathcal{M}$. In this picture, stemming from the discrete character of $\mathcal{M}$, motion along a trajectory defined by a vector field $X$, is constrained to be along arrows of the di-graph, so that at each point $i$ of $\mathcal{M}$ (i.e. vertex of the di-graph), motion along any arrow is either certain or impossible, depending on $X$.

The above discussion raises naturally the question what kind of "motion" is described by an arbitrary vector field, i.e. one not necessarily satisfying (3.1X)? Evidently, $\left\{e_{i}\right\}$ (equivalently, $\mathcal{M}$ ) is no longer mapped onto itself, but to a set of nontrivial linear combinations of the $e_{i}$ 's, that is, to a "superposition of points" (cf. [7], §3). This reminds the situation in statistical mechanics; non-unitary semigroups of operators on the state space, transform pure states into mixtures in the quantum case and $\delta$-distributions into more general ones in the classical case.

On the basis of this analogy and the discussion in the previous paragraph, we shall interpret a vector field $X$ as the generator of evolution on $\mathcal{M}$, having at each point a particular 
well-defined probability associated with each arrow that emerges from that point. More precisely, in this interpretation, the component $X^{i j}$ of $X$ gives the transition probability for the "infinitesimal" motion from $i$ to $j$.

In this picture, vector fields $X$ acquire a double role; as generators of evolution and as states giving the transition probabilities for "infinitesimal" changes. This double role is reflected in the notation of (3.9) so that $\langle\mathrm{d} f, X\rangle=X(f)$ is the expectation (average) value for the "infinitesimal" change of the observable $f$ along $X$ (cf. (3.14)). It is a key idea following from adopting a discrete structure as our starting point. More precisely, though both in the discrete and the usual continuous case, vector fields are linear combinations of 1st order difference and differential operators, respectively, it is only in the continuous case that any such combination generates automorphisms of the algebra of functions, or equivalently, flows of trajectories (i.e. what we call here a deterministic evolution, or motion). This is due to the fact that, in contrast to the continuum, nontrivial linear combinations of displacements do not give a permissible displacement on the di-graph. In the rest of this section we shall pursue further this idea.

\subsection{The oriented hypercubic lattice and vector fields on it}

To be more specific and for applications in subsequent sections, we shall henceforth take $\mathcal{M}$ to be an oriented hypercubic lattice; that is, $\mathcal{M}=\mathbb{Z}^{N}$ with elements $\vec{k} \in \mathcal{M}$ and

$$
e_{\vec{k} \vec{l}} \neq 0 \quad \Leftrightarrow \quad \vec{l}=\vec{k}+\hat{\mu}, \quad \hat{\mu}:=\left(\delta_{\mu}^{\nu}\right)
$$

$\{\hat{\mu}\}$ being the canonical basis of $\mathbb{R}^{N}$ (for details see [11]). Then, by defining

$$
u^{\mu}:=\sum_{\vec{k}} k^{\mu} e_{\vec{k}}
$$

and writing $u:=\left(u^{\mu}\right)$, we get for $f \in \mathcal{A}$

$$
f=\sum_{\vec{k}} f(\vec{k}) e_{\vec{k}}=f(u)
$$

so that from (3.6), (3.14) we get

$$
\begin{gathered}
\mathrm{d} u^{\mu}=\sum_{\vec{k}} e_{\vec{k}, \vec{k}+\hat{\mu}} \\
\mathrm{d} f=\sum_{\vec{k}, \mu}(f(\vec{k}+\hat{\mu})-f(\vec{k})) e_{\vec{k}, \vec{k}+\hat{\mu}}=\sum_{\mu}(f(u+\hat{\mu})-f(u)) \mathrm{d} u^{\mu} \\
=: \sum_{\mu}\left(\partial_{+u^{\mu}} f\right) \mathrm{d} u^{\mu} \\
\partial_{+u^{\mu}}=\sum_{\vec{k}} \partial_{\vec{k}, \vec{k}+\hat{\mu}}
\end{gathered}
$$


where we use the same symbol for $f$ as a function of $\vec{k}$ and of $u$, to avoid a cumbersome notation.

Clearly, $\left\{\mathrm{d} u^{\mu}\right\}$ is a basis for 1-forms with dual basis for vector fields $\left\{\partial_{+u^{\mu}}\right\}$

$$
\begin{gathered}
X=\sum_{\vec{k}, \mu} X^{\vec{k}, \vec{k}+\hat{\mu}} \partial_{\vec{k}, \vec{k}+\hat{\mu}}=: \sum_{\mu} P^{\mu} \partial_{+u^{\mu}}, \quad P^{\mu}=\sum_{\vec{k}} X^{\vec{k}, \vec{k}+\hat{\mu}} e_{\vec{k}} \\
\left\langle\mathrm{~d} u^{\mu}, \partial_{+u^{\nu}}\right\rangle=\delta_{\nu}^{\mu}, \quad \mathrm{d} u^{\mu} \bullet \mathrm{d} u^{\nu}=\delta^{\mu \nu} \mathrm{d} u^{\mu}
\end{gathered}
$$

the last relation following from (3.6).

The interpretation of vector fields as states giving transition probabilities, discussed in the previous subsection is manifested in (3.24); $X^{\vec{k}, \vec{k}+\hat{\mu}}$ is the transition probability at a point $\vec{k}$ in the direction of the axis $u^{\mu}$, so that $P^{\mu}$ is the distribution of this probability on $\mathcal{M}$. Therefore, from now on we consider vector fields $X$ such that

$$
P^{\mu} \geq 0, \quad \sum_{\mu} P^{\mu}=1
$$

Thus, in view of (3.22), (3.25), $\langle\mathrm{d} f, X\rangle$ gives at each point of $\mathcal{M}$, the expectation of $\mathrm{d} f$ (the "infinitesimal" change of $f$ ), along $X$, with respect to the probability distribution determined by $X$. To see that this interpretation is consistent and for later use as well, we notice the following: The l.h.s. of (3.17ii) is rewritten for an arbitrary $X=\sum_{\mu} P^{\mu} \partial_{+u^{\mu}}$ as

$$
P^{\mu \nu}:=\delta^{\mu \nu} P^{\mu}-P^{\mu} P^{\nu}=\left\langle\mathrm{d} u^{\mu} \bullet \mathrm{d} u^{\nu}, X\right\rangle-\left\langle\mathrm{d} u^{\mu}, X\right\rangle\left\langle\mathrm{d} u^{\nu}, X\right\rangle,
$$

where (3.25) has been used. $\left(P^{\mu \nu}\right)$ is the correlation matrix of the $N$-dimensional random variable $\left(\mathrm{d} u^{\mu}\right)$ having a probability distribution (determined by) $X$, where $\bullet$ is the natural (intrinsic) product of 1-forms. $P^{\mu \nu}$ vanishes if and only if $X$ generates a flow of trajectories in $\mathcal{M}$. Actually, at $\vec{k} \in \mathcal{M}, P^{\mu \nu}(\vec{k})$ is the correlation matrix of the following random variables

$$
I_{\mu}(\vec{k})= \begin{cases}1 & \text { if change at } \vec{k} \text { is along the } u^{\mu} \text { axis } \\ 0 & \text { otherwise }\end{cases}
$$

with probabilities $P^{\mu}(\vec{k})$ (cf. [37], $\S 3.3$, p.331).

The following proposition has an interesting probabilistic interpretation to be used later on

Proposition 3.3 For $\omega=\sum_{\mu} s_{\mu} \mathrm{d} u^{\mu} \in \Omega^{1}$, write $s^{t}=\left(s_{\mu}\right)$ (row vector). Then

$$
\langle\omega \bullet \omega, X\rangle-(\langle\omega, X\rangle)^{2}=s^{t} \mathbb{P} s
$$

with $\mathbb{P}=\left(P^{\mu \nu}\right)$. Moreover, for

$$
\rho:=\sum_{\mu} \mathrm{d} u^{\mu}=\sum_{\vec{k}, \mu} e_{\vec{k}, \vec{k}+\hat{\mu}}
$$


we have

$$
\begin{gathered}
\rho \bullet \omega=\omega, \quad \forall \omega \in \Omega^{1} \\
\langle\rho \bullet \rho, X\rangle-(\langle\rho, X\rangle)^{2}=0, \quad\langle\rho \bullet \omega, X\rangle-\langle\rho, X\rangle\langle\omega, X\rangle=0, \quad \forall \omega \in \Omega^{1} \\
P^{\mu \nu}=\left\langle\left(\mathrm{d} u^{\mu}-\left\langle\mathrm{d} u^{\mu}, X\right\rangle \rho\right) \bullet\left(\mathrm{d} u^{\nu}-\left\langle\mathrm{d} u^{\nu}, X\right\rangle \rho\right), X\right\rangle
\end{gathered}
$$

The proof is immediate, but we stress the fact that, for (3.31) it is essential that $\sum_{\mu} P^{\mu}=1$.

$\mathrm{Eq}(3.32)$ is an equivalent expression of (3.27), obtained by using the unit $\rho$ with respect to the natural multiplication - of 1-forms. On the other hand, in the present conceptual framework, (3.28) gives the variance of the random variable $\left(s_{\mu}\right)$ (and for that matter, of $\omega$ ) having probability distribution $X$. Thus (3.31) says that the unit of $(\Omega, \bullet)$ has zero variance and is uncorrelated with all $\omega \in \Omega^{1}$. Thus, as a random variable, $\rho$ is constant, a fact that will be used in the next subsection. Algebraically, (3.31 ii) says that $\mathbb{P}$ has always a zero eigenvalue and a corresponding 1 D-eigenspace $\{\lambda(1,1, \ldots, 1), \lambda \in \mathbb{R}\}$. It is easily seen that this is the only generic eigenspace of zero, in the sense that it is the only one existing at all points of $\mathcal{M}$.

\subsection{The concept of time}

As already mentioned at the beginning of this section, we consider $\mathcal{M}$ to be the extended phase space, in the sense that it contains the "time axis". Adopting a newtonian picture we require:

(a) For every evolution (e.g. motion) in $\mathcal{M}$, a change of time is required;

(b) Time flows with certainty, that is, there is always a change in the time axis.

Then, if any evolution on $\mathcal{M}$ is determined by some vector field $X$, then (a) means that the time change is given by 1 -forms $\tau$ such that $\left\langle\tau, \partial_{+u^{\mu}}\right\rangle \neq 0$ at all points of $\mathcal{M}$. Moreover, in view of the discussion following proposition 3.3 , (b) implies that

$$
\tau=b \rho=\mathrm{d}\left(-b \sum_{\mu} u^{\mu}\right)=: \mathrm{d} t, \quad\langle\mathrm{~d} t, X\rangle=-b, \quad \mathrm{~d} t \bullet \mathrm{d} t=-b \mathrm{~d} t, \quad b \in \mathbb{R}^{+}
$$

so that (a) is also satisfied.

(i) Since $u^{\mu}, P^{\mu}$ are dimensionless, $b$ is put for dimensional reasons.

(ii) By the interpretation of vector fields $X$ both as generators of evolution and states, $X(f)=0$ should describe the evolution of observables in the extended phase space. Then, as explained in appendix A.2, $X(t)$ should be negative, in analogy with classical dynamics, hence the choice of the $(-)$ sign in (3.33).

\subsection{General prescription of dynamical evolution}

In summary, the approach described in this section leads to a general prescription for time evolution in $\mathcal{M}$ : 
- $\mathcal{M}$ is taken to be the extended phase space (cf. §3.4).

- Via (3.9), vector fields $X$ are seen both as generators of evolution and as states giving the transition probabilities for "infinitesimal" changes (cf. $\S \S 3.2,3.3$ ).

- Time change appears in steps of duration $b$.

Therefore, elements $f$ of $\mathcal{A}$ are observables, whose "infinitesimal" change has an expectation value with respect to $X,\langle\mathrm{~d} f, X\rangle=X(f)$. But then, by remark (ii) in $\S 3.4$ and appendix A.2, $-X(f) / b$ gives the rate of change of $f$ along $X$ in extended phase space, hence, in analogy with classical dynamics, we take as the general dynamical evolution equation

$$
\text { evolution equation for observable } f: \quad-\frac{X(f)}{b}=0
$$

which becomes with the aid of (3.22), (3.24)

$$
-\frac{1}{b} \sum_{\mu} P^{\mu}(u)(f(u+\hat{\mu})-f(u))=0
$$

$\mathrm{Eq}(3.34)$ is a general prescription, independent of the choice of $\mathcal{M}$ as an oriented hypercubic lattice. Nevertheless, it is our starting point in the applications in the next sections. As we shall see there, in the continuous limit, (3.35) reduces in particular cases to the adjoint of well known kinetic equations, i.e. to evolution equations for observables.

\section{One Dimensional Problems}

It is quite straightforward to apply the general ideas and results of $\S 3$ to specific cases. To keep technical details to a minimum, we consider in this section as an illustration, the derivation of well known 1D-model evolution equations and leave the general treatment to the next sections.

Motivated by $\S 3.4$ and the model of $\S 2$, we consider a coordinate system $(t, x)$ in the notation of $\S 2$. Although the transformation from the lattice axes to $(t, x)$ can be any invertible transformation, we postpone this till the next section and use "light-cone" coordinates defined by (2.4). Then, for $f \in \mathcal{A}, \mathrm{d} f$ is given by (2.7). On the other hand, (3.24) is

$$
\begin{aligned}
X & =p \partial_{+u}+q \partial_{+v}=\langle\mathrm{d} u, X\rangle \partial_{+u}+\langle\mathrm{d} v, X\rangle \partial_{+v} \\
& =\left(\sum_{\vec{k}} X^{\vec{k}, \vec{k}+\hat{1}} e_{\vec{k}}\right) \partial_{+u}+\left(\sum_{\vec{k}} X^{\vec{k}, \vec{k}+\hat{2}} e_{\vec{k}}\right) \partial_{+v},
\end{aligned}
$$

where $p, q \geq 0$ and $p+q=1$. Using (2.7) in (3.34) and that $p+q=1$, we readily obtain the evolution equation

$$
\begin{aligned}
-\frac{X(f)}{b} & =\left(\partial_{-t} f(t, x)-\frac{h}{2} \Delta_{x} f(t-b, x)\right) \frac{\langle\mathrm{d} t, X\rangle}{-b}+\bar{\partial}_{x} f(t-b, x) \frac{\langle\mathrm{d} x, X\rangle}{-b} \\
& =\left(\partial_{-t} f(t, x)-\frac{h}{2} \Delta_{x} f(t-b, x)\right)-\frac{a}{b}(p-q) \bar{\partial}_{x} f(t-b, x)=0
\end{aligned}
$$


In the derivation of (4.2), we remark that the following features are present in any number of dimensions (see e.g. $\S 5$ and appendix B):

(a) Because of $(3.26),\langle\mathrm{d} t, X\rangle=-b$.

(b) 2nd order difference operators appear only in the coefficient of $\mathrm{d} t$.

(c) the term involving $\langle\mathrm{d} x, X\rangle=X(x)$ has the form of a drift term.

More precisely, $\langle\mathrm{d} x, X\rangle$ has been interpreted in $\S 3.5$ as the "infinitesimal" change of $x$ in one step of time $b$, hence it is natural to assume that it is proportional to $b$. This assumption can be justified on the basis of the general approach described in $\S 5$ (see (5.13), the derivation of (5.15) and appendix A.3). Since we eventually pass to the continuous limit considered in $\S 2$, namely $a, b \rightarrow 0, a^{2} / b \rightarrow h=$ constant, we have

$$
\langle\mathrm{d} x, X\rangle=a(p-q)=o(b) \quad \Rightarrow \quad p-q=o\left(\frac{a}{h}\right)=o(a)
$$

and therefore

$$
\frac{\langle\mathrm{d} x, X\rangle}{b}=\frac{a}{b}(p-q) \rightarrow R
$$

say, when $a, b \rightarrow 0, a^{2} / b \rightarrow h$, so that in this limit, (4.2) becomes

$$
\partial_{t} f-R \partial_{x} f-\frac{h}{2} \partial_{x}^{2} f=0
$$

To see the physical meaning of this evolution equation, we go back to (3.24) and consider two special cases.

1. $X^{\vec{k}, \vec{k}+\hat{1}}, X^{\vec{k}, \vec{k}+\hat{2}}$ are constant:

Then from (4.1), (4.3)

$$
X^{\vec{k}, \vec{k}+\hat{1}}=p=: \frac{1}{2}-\gamma a, \quad X^{\vec{k}, \vec{k}+\hat{2}}=q=: \frac{1}{2}+\gamma a, \quad \gamma=\text { constant }
$$

As it is evident from the figure, $p, q$ represent the transition probability for right and left motion respectively, along the $x$-axis. This is a random walk model in 1 D-position space and in view of (4.2), the evolution equation in the continuous limit, is

$$
\partial_{t} f+2 \gamma h \partial_{x} f-\frac{h}{2} \partial_{x}^{2} f=0
$$

This is the adjoint of Smoluchowski's equation for a constant force field, of intensity proportional to $\gamma$ and diffusion constant $h$, derived here in a conceptual framework totally different from that conventionally used (e.g. the theory of Markov processes; see [31], §3).

2. Evolution in velocity space.

Here $x$ represents the velocity of a particle and we take (cf. (4.3))

$$
X^{\vec{k}, \vec{k}+\hat{1}}=\frac{1}{2}-\beta b\left(k^{1}-k^{2}\right), \quad X^{\vec{k}, \vec{k}+\hat{2}}=\frac{1}{2}+\beta b\left(k^{1}-k^{2}\right), \quad \beta=\text { constant }>0
$$

hence by (3.24), (2.4)

$$
p=\frac{1}{2}-\beta b(u-v), \quad q=\frac{1}{2}+\beta b(u-v), \quad p-q=-2 \frac{b}{a} \beta x
$$




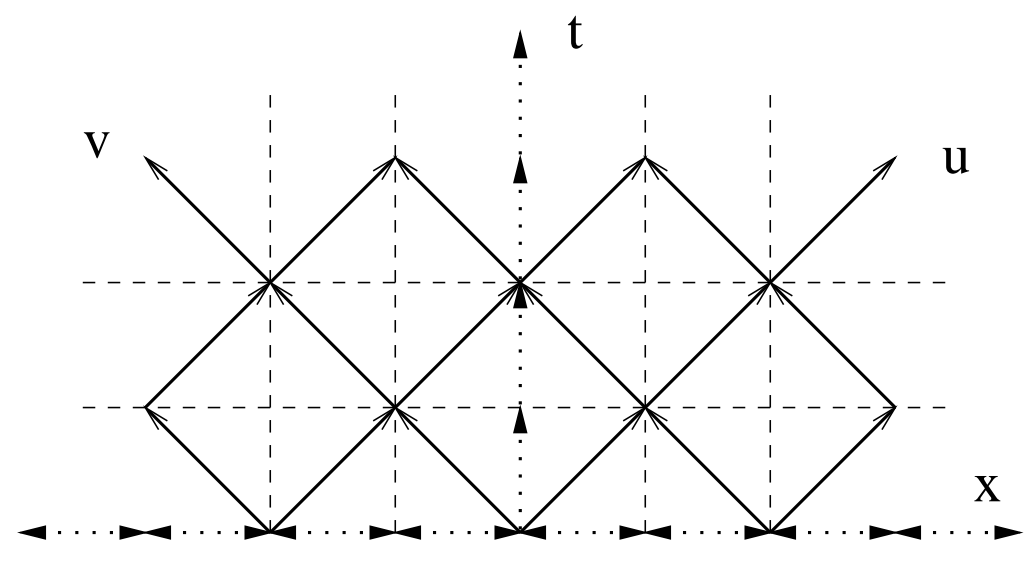

Coordinate transformation on the square lattice

so that in the continuous limit, (4.2) gives

$$
\partial_{t} f+2 \beta x \partial_{x} f-\frac{h}{2} \partial_{x}^{2} f=0
$$

This is the adjoint to the 1D FP equation in velocity space, giving the Ornstein-Uhlenbeck process (diffusion constant $h$, drift coefficient $\beta$ ). We may remark however, that if $x$ is interpreted as a position coordinate, then (4.10) is the adjoint to the Smoluchowski equation for a harmonic external field (cf. [31], §4).

\section{Evolution Equation: The General Case}

In this section we apply the results of $\S 3$ in the case of an $(N+1)$-oriented hypercubic lattice $\mathcal{M}$.

The lattice coordinates $u^{\mu}$ are dimensionless. We consider linear coordinate transformations to physical coordinates

$$
x^{\mu}=a_{\mu} \sum_{\nu} A_{\nu}^{\mu} u^{\nu}, \quad \mu, \nu=0,1, \ldots, N
$$

in which $a_{\mu}$ are scaling parameters with the dimensions of $x^{\mu}$. In view of $\S 3.4$ we require that

$$
x^{0}=t, \quad \text { hence } \quad a_{0}=-b, \quad A_{\mu}^{0}=1
$$

and we shall eventually consider the continuous limit

$$
a_{\mu} \rightarrow 0, \quad \frac{a_{i} a_{j}}{b} \rightarrow h_{i j}, \quad \frac{a_{i}}{b} \rightarrow \pm \infty, \quad A_{\nu}^{\mu} \rightarrow \hat{A}_{\nu}^{\mu}
$$

with the $A_{\nu}^{\mu}$ depending on the $a_{\mu}$ 's, in general. $\mathrm{Eq}(5.3)$ is motivated by the limiting procedure considered in [13, 15] and $\S 2$ here. Nevertheless, its nature is further explored in $\S 8$. Here we only notice that, in contrast to $\left(t, x^{i}\right)$, the $u^{\mu}$ are not defined in this limit. Moreover, to avoid a too technical development, we restrict the discussion to linear transformations with 
constant coefficients, thus making the physical and conceptual aspects of our approach more clear. In what follows, latin indices run from 1 to $N$, greek ones from 0 to $N$ and symbols with a hat denote quantities in the limit (5.3).

By (5.1) we can write

$$
u^{\mu}=\sum_{\nu} B_{\nu}^{\mu} \frac{x^{\nu}}{a_{\nu}}, \quad \sum_{\nu} A_{\nu}^{\mu} B_{\rho}^{\nu}=\delta_{\rho}^{\mu}, \quad B_{\nu}^{\mu} \rightarrow \hat{B}_{\nu}^{\mu}
$$

so that (3.22), (3.25) become with the aid of (2.9)

$$
\mathrm{d} f=\left(\partial_{-t} f(t, x)-\frac{1}{2} \Delta f(t-b, x)\right) \mathrm{d} t+\sum_{i} \bar{\partial}_{i} f(t-b, x) \mathrm{d} x^{i}
$$

where

$$
\begin{aligned}
\bar{\partial}_{i} f(t, x) & :=\frac{1}{a_{i}} \sum_{\mu} f\left(t, x^{j}+a_{j} A_{\mu}^{j}\right) B_{i}^{\mu}, \\
\Delta f(t, x) & :=\frac{2}{b}\left(\sum_{\mu} f\left(t, x^{j}+a_{j} A_{\mu}^{j}\right) B_{0}^{\mu}-f(t, x)\right)
\end{aligned}
$$

and

$$
\begin{gathered}
\mathrm{d} t \bullet \mathrm{d} t=-b \mathrm{~d} t, \quad \mathrm{~d} t \bullet \mathrm{d} x^{i}=-b \mathrm{~d} x^{i} \\
\mathrm{~d} x^{i} \bullet \mathrm{d} x^{j}=-\frac{a_{i} a_{j}}{b} \sum_{\mu} A_{\mu}^{i} A_{\mu}^{j} B_{0}^{\mu} \mathrm{d} t+\sum_{\mu, m} \frac{a_{i} a_{j}}{a_{m}} A_{\mu}^{i} A_{\mu}^{j} B_{m}^{\mu} \mathrm{d} x^{m}
\end{gathered}
$$

With the interpretation of vector fields $X$ given in $\S \S 3.2,3.3$, we consider those $X$ that satisfy (3.26), i.e.

$$
X=\sum_{\mu} P^{\mu} \partial_{+u^{\mu}}, \quad P^{\mu} \geq 0, \quad \sum_{\mu} P^{\mu}=1
$$

hence $\langle\mathrm{d} t, X\rangle=-b,\left\langle\mathrm{~d} u^{i}, X\right\rangle=P^{i}$. Therefore the evolution equation (3.34) becomes (c.f. (4.2)

$$
-\frac{1}{b} X(f)=\partial_{-t} f(t, x)-\frac{1}{2} \Delta f(t-b, x)-\sum_{i} \frac{a_{i}}{b}(A P)^{i} \bar{\partial}_{i} f(t-b, x)=0
$$

where, by (5.1)

$$
\left\langle\mathrm{d} x^{i}, X\right\rangle=a_{i}(A P)^{i}
$$

With the interpretation of vector fields introduced in $\S 3$, this is the average value of the "infinitesimal" change of $x^{i}$ along $X$ in one time-step $b$. By assuming that $f$ is sufficiently well-behaved function, Taylor expansion of (5.10) around $x^{i}$, gives

$$
-\frac{1}{b} X(f)=\partial_{t} f-\sum_{i} \frac{a_{i}}{b}(A P)^{i} \partial_{i} f-\frac{1}{2} \sum_{i, j} \frac{a_{i} a_{j}}{b}\left(\sum_{\mu} A_{\mu}^{i} A_{\mu}^{j} P^{\mu}\right) \partial_{i} \partial_{j} f+o(a)=0
$$


We proceed further in analogy with conventional dynamics. Let $\left(R^{i}\right)$ be the generator of motion in this case (e.g. in Newton's or Hamilton's equations). Then, as it is explained in appendix A.2, to the system of characteristic equations of the observables' evolution equation (A.3iii), are associated the 1-forms

$$
\alpha^{i}=\mathrm{d} x^{i}+R^{i} \mathrm{~d} t
$$

which vanish along the integral curves of the vector field that gives the dynamical evolution.

Therefore, in the present discrete context, we postulate the existence of dynamics in the above sense, that is, the generator of dynamical evolution is determined by a similar relation, namely

$$
\left\langle\alpha^{i}, X\right\rangle=\left\langle\mathrm{d} x^{i}, X\right\rangle+R^{i}\langle\mathrm{~d} t, X\rangle=0 \quad \text { hence } \quad a_{i}(A P)^{i}=b R^{i}
$$

(cf. (A.5). Hence,

$$
(A P)^{i}=\frac{b}{a_{i}} R^{i}=\frac{a_{i}}{h_{i i}} R^{i}+o(a) \quad \text { with } \quad R^{i} \rightarrow \hat{R}^{i}
$$

Notice that the postulated existence of dynamics, that is (5.14i), implies that $\left\langle\mathrm{d} x^{i}, X\right\rangle$ is $o(b)$, thus justifying this assumption which was introduced in the derivation of (4.4). It is also equivalent to a similar relation determining the transition probabilities $P^{\mu}$, as explained in appendix A.3.

Since $(A P)^{0}=1$ (because of (5.2), (5.9)), we can invert (5.15) to get

$$
P^{\mu}=B_{0}^{\mu}+\sum_{m} \frac{a_{m}}{h_{m m}} B_{m}^{\mu} R^{m}, \quad \text { hence in the limit } \quad \hat{P}^{\mu}=\hat{B}_{0}^{\mu}
$$

so that by (5.9)

$$
\sum_{\mu} \hat{B}_{0}^{\mu}=1, \quad \hat{B}_{0}^{\mu} \geq 0
$$

Therefore (5.12) can be rewritten as

$$
-\frac{1}{b} X(f)=\partial_{t} f-\sum_{i} \frac{a_{i}^{2}}{h_{i i} b} R^{i} \partial_{i} f-\frac{1}{2} \sum_{i, j} \eta^{i j} \partial_{i} \partial_{j} f+o(a)=0
$$

where

$$
\eta^{i j}:=\frac{a_{i} a_{j}}{b} \sum_{\mu} A_{\mu}^{i} A_{\mu}^{j} B_{0}^{\mu} \quad \rightarrow \quad \hat{\eta}^{i j}=h_{i j} \sum_{\mu} \hat{A}_{\mu}^{i} \hat{A}_{\mu}^{j} \hat{P}^{\mu}
$$

In the continuous limit (5.3), we have

$$
\partial_{t} f-\sum_{i} \hat{R}^{i} \partial_{i} f-\frac{1}{2} \sum_{i, j} \hat{\eta}^{i j} \partial_{i} \partial_{j} f=: \hat{X} f=0
$$


This is our general evolution equation for observables $f$, corresponding to the DC obtained from $(5.8)$ in the limit $(5.3)$

$$
\mathrm{d} t \bullet \mathrm{d} t=0, \quad \mathrm{~d} t \bullet \mathrm{d} x^{i}=0, \quad \mathrm{~d} x^{i} \bullet \mathrm{d} x^{j}=-\hat{\eta}^{i j} \mathrm{~d} t
$$

$\mathrm{Eq}(5.21)$ is identical with the DC considered in [13, 15], called 2nd order DC there, because it leads to 2 nd order evolution equations, i.e. (5.20). We shall come to this point again in $\S 9$.

A number of interesting remarks can be made here:

1) If (5.17) holds, then $\hat{\eta}^{i j}$ is a non-negative definite matrix, so that (5.20) is the adjoint of a generalized FP-equation with diffusion matrix $\hat{\eta}^{i j}$ and drift vector $\hat{R}^{i}$.

2) Under the transformation (5.1), the correlation matrix of the lattice, (3.27), becomes

$$
\begin{gathered}
H^{\mu \nu}:=\left(A \mathbb{P} A^{t}\right)^{\mu \nu} \\
H^{\mu 0}=0 \\
H^{i j}=\sum_{\mu} a_{i} a_{j} A_{\mu}^{i} A_{\mu}^{j} P^{\mu}-a_{i} a_{j}(A P)^{i}(A P)^{j}=\left\langle\mathrm{d} x^{i} \bullet \mathrm{d} x^{j}, X\right\rangle-\left\langle\mathrm{d} x^{i}, X\right\rangle\left\langle\mathrm{d} x^{j}, X\right\rangle \\
=\left\langle\mathrm{d}\left(x^{i} x^{j}\right)-x^{i} \mathrm{~d} x^{j}-x^{j} \mathrm{~d} x^{i}, X\right\rangle-\left\langle\mathrm{d} x^{i}, X\right\rangle\left\langle\mathrm{d} x^{j}, X\right\rangle
\end{gathered}
$$

where we have used the Leibniz rule (1.6).

- $H^{\mu 0}=0$ reflects the fact that the time change $\mathrm{d} t$ is the (essentially) unique eigenvector of zero of $\mathbb{P}$, as discussed in $\S 3.4$

- Because of (5.15), the last term on the r.h.s of (5.23) is $o\left(a_{i}{ }^{4}\right)$.

- Using the unit $\rho$ of the algebra $\left(\Omega^{1}, \bullet\right)$, eq(3.29), we may write

$$
H^{i j}=\left\langle\left(\mathrm{d} x^{i}-\left\langle\mathrm{d} x^{i}, X\right\rangle \rho\right) \bullet\left\langle\left(\mathrm{d} x^{j}-\left\langle\mathrm{d} x^{j}, X\right\rangle \rho\right), X\right\rangle\right.
$$

(cf.(3.32)). In fact, $H^{i j}$ is the correlation matrix of the 1 -forms $\alpha^{i}$, eq(5.13), associated with the equations of motion via (5.14)

$$
\begin{aligned}
H^{i j} & =\left\langle\left(\alpha^{i}-\left\langle\alpha^{i}, X\right\rangle \rho\right) \bullet\left\langle\left(\alpha^{j}-\left\langle\alpha^{j}, X\right\rangle \rho\right), X\right\rangle\right. \\
& =\left\langle\alpha^{i} \bullet \alpha^{j}, X\right\rangle-\left\langle\alpha^{i}, X\right\rangle\left\langle\alpha^{j}, X\right\rangle \\
& =\left\langle\alpha^{i} \bullet \alpha^{j}, X\right\rangle
\end{aligned}
$$

Therefore in the continuous limit we get

$$
\begin{aligned}
\frac{H^{i j}}{b} \rightarrow \hat{\eta}^{i j} & =\left\langle\mathrm{d} x^{i} \bullet \mathrm{d} x^{j}, \hat{X}\right\rangle=\left\langle\mathrm{d}\left(x^{i} x^{j}\right)-x^{i} \mathrm{~d} x^{j}-x^{j} \mathrm{~d} x^{i}, \hat{X}\right\rangle \\
& =\hat{X}\left(x^{i} x^{j}\right)-x^{i} \hat{X}\left(x^{j}\right)-x^{j} \hat{X}\left(x^{i}\right)
\end{aligned}
$$

Thus, $\hat{\eta}^{i j}$ is essentially the limiting value of (the matrix of) correlations of "infinitesimal" changes along the axes of the lattice, induced by the generator of evolution $X$. In fact, by (5.22), $\hat{\eta}^{i j}$ vanishes if $X$ is the generator of a deterministic flow on the lattice, i.e. when the 
concept of a trajectory is well-defined for $X$, as explained in $\S 3.3$ (cf.(3.17)). This is reflected in the limiting equation (5.25), which gives the deviation of $\hat{X}$ from an ordinary derivation, that is, an ordinary vector field for which the concept of a trajectory is (locally) always meaningful. We may also compare $\hat{\eta}^{i j}$ with the usual interpretation of the diffusion matrix in the kinetic theory of open systems. There, it is the correlation matrix of the hamiltonian vector field of the interaction hamiltonian of the open system with the bath, with respect to the state of the bath (see e.g. 38] eq(4.16)). A similar interpretation of this quantity exists in the theory of markovian stochastic processes and of Itô's stochastic calculus (e.g. [39] chs.3, 4, [40], ch.VIII).

On the other hand, the above comments provide an interpretation of the noncommutativity of the DC defined by (5.21) and studied in previous works ([13, 15]): Starting with a discrete structure of the phase-space, somehow reflects the fact that originally one considers the fine microscopic structure of the system. At this level, changes are described by structures that are necessarily noncommutative and it is a generic characteristic of vector fields in this case, not to describe deterministic motion (i.e. flows of trajectories), in contrast with vector fields in the (ordinary) continuous case for which this is always the case. Then, the passage to the continuous limit corresponds to a coarse-graining process, in which the fine details of the micro-structure are no longer explicit, but which, nevertheless, have been taken into account. In particular, the above generic characteristic of vector fields on a discrete phase-space is expressed quantitatively in the continuous limit, by the non-vanishing of $\hat{\eta}^{i j}$, eq(5.19).

3) In 1) above, it was mentioned that $\left(\hat{\eta}^{i j}\right)$ is nonnegative-definite (being the limit of such matrices). On the other hand it is an easy matter to see that for such a matrix, $\hat{\eta}^{i i}=0$ for some fixed $i$ implies $\hat{\eta}^{i j}=0$ for all $j$. In our case, this means that with respect to $x^{i}, \hat{X}$ in $(5.20)$ is a derivation, hence it describes deterministic motion along the $x^{i}$-axis.

4) The limiting eq( $(5.20)$ is the most general linear autonomous differential (evolution) equation, whose adjoint admits a probabilistic interpretation; more precisely, under mild regularity conditions on the coefficients, it defines a positivity and normalization preserving, strongly continuous semigroup, globally on the space of (continuous) observables $f$ having a finite limit at infinity. Its adjoint also defines a positivity and normalization preserving semigroup, satisfying a general $H$-theorem ([30], theorems 5.1,6.1). This is assured by the non-negative definiteness of $\hat{\eta}^{i j}$. In our case this follows from (5.17), which is a consequence of the interpretation of a vector field as a transition probability-distribution for infinitesimal changes. Thus, if $\left\{\tilde{\partial}_{t}, \tilde{\partial}_{x^{i}}\right\}$ is the basis of vector fields dual to $\left\{\mathrm{d} t, \mathrm{~d} x^{i}\right\}$, then

$$
B_{0}^{\mu}=\left\langle\mathrm{d} u^{\mu},-b \tilde{\partial}_{t}\right\rangle=-b \tilde{\partial}_{t}\left(u^{\mu}\right) \quad \rightarrow \quad \hat{B}_{0}^{\mu} \geq 0
$$

Therefore, $\hat{B}_{0}^{\mu} \geq 0$ means that in the limit of the continuum (i.e. in a macroscopic description - see 2) above), changes in phase-space are in the future direction (cf. the discussion following eq(A.3) in Appendix A). Thus, in this picture, the "macroscopic" direction of time is intimately related to a probabilistic interpretation of evolution at the "microscopic" level (here the terms "macroscopic" and "microscopic" correspond to the terms "continuous description" and "discrete description" in the sense of 2) above).

\footnotetext{
${ }^{5}\left(\hat{\eta}^{i j}\right)$ defines a (possibly degenerate) nonnegative-definite scalar product on $\mathbb{R}^{N}$, and we apply the Schwartz inequality to the $i$-th element of the canonical basis of $\mathbb{R}^{N}$ with each $j$-th element $j \neq i$.
} 
5) The expansion of the evolution equation (3.34) (or (5.10)) in powers of the $a_{i}$ is not essential, but has been done because of the generality of the transformation (5.1). In each particular case $X(f)$ may be written explicitly in terms of appropriate difference operators, which in the continuous limit yield the corresponding FP equation (e.g. as in $\S 4$ ). Such an $N$-dimensional example is worked out in Appendix B.

\section{Transformations in phase-space}

There is considerable freedom in the choice of the coordinates $\left(x^{i}\right)$, eq(5.1), in such a way that the continuous limit exists. In this section we comment briefly on this issue.

Let $\left(x^{\mu}\right),\left(x^{\prime \mu}\right)$ be coordinate systems on $\mathcal{M}$, obtained from the lattice coordinates by a linear transformation like eq(5.1) and let $x^{\prime \mu}=\Lambda_{\nu}^{\mu} x^{\nu}$. In view of the discussion in $\S \S 3.3,3.4$, 5 , from a physical point of view we require that $\Lambda$ leaves $\mathrm{d} t$ invariant. Then it has the form

$$
\Lambda=\left(\begin{array}{c|c}
1 & \mathbf{0} \\
\hline \Lambda^{i} & \Lambda_{j}^{i}
\end{array}\right)
$$

Such transformations form a group. Moreover, since $\mathrm{d} t$ is in the kernel of the correlation matrix $\left(H^{\mu \nu}\right)$, eq(5.22), $\Lambda$ leaves the form of $H^{\mu \nu}$ (i.e. $H^{\mu 0}=0$ ) unaltered:

$$
H=\left(\begin{array}{c|c}
0 & \mathbf{0} \\
\hline 0 & H^{i j}
\end{array}\right) \quad \stackrel{\Lambda}{\longrightarrow} \quad H^{\prime}=\left(\begin{array}{c|c}
0 & \mathbf{0} \\
\hline 0 & \left(\Lambda H \Lambda^{t}\right)^{i j}
\end{array}\right)
$$

If we write (5.1) in the form

$$
\left(u^{\mu}\right) \stackrel{A}{\longrightarrow}\left(\begin{array}{l}
x^{\mu} \\
\hline a_{\mu}
\end{array}\right): \quad A=\left(\begin{array}{c|c}
1 & \mathbf{1} \\
\hline A^{i} & A_{j}^{i}
\end{array}\right)
$$

where $\mathbf{1}=(1,1, \ldots, 1)$, then

$$
H^{\prime}=(\Lambda A) \mathbb{P}(\Lambda A)^{t}
$$

A natural (partial) fixing of the coordinate freedom is to choose $\Lambda$ so that the correlation matrix, hence $\hat{\eta}^{i j}$ in (5.20), is diagonal. However many other possibilities exist, see e.g. Appendix B and section 7 .

\section{Further Applications: 2D Evolution Equations}

As a concrete application of the results of $\S 5$, which also exhibits the main features of the general $N$-dimensional case, we consider in this section the general form of the evolution equation (5.20) in two dimensions. Then, we show how evolution equations that are used in statistical physics as model kinetic equations can be derived in the present context, at the same time throwing some light into their foundations from a new perspective. 
An example of this type is Kramers' equation which for simplicity is considered here in one spatial dimension $x$ :

$$
\partial_{t} \rho+y \partial_{x} \rho+F(x) \partial_{y} \rho-\beta \partial_{y}(y \rho)-\frac{h}{2} \partial_{y}^{2} \rho=0
$$

This is a kinetic equation for the probability distribution $\rho$ of a (unit mass) brownian particle of velocity $y$ in an equilibrium bath of temperature $h / 2 \beta, \beta$ being its friction (drift) coefficient and $F$ an external force field.

$\mathrm{Eq}(7.1)$ can be derived from microscopic dynamics, e.g. by expanding the so-called generalized master equation in powers of the ratio of the masses of the bath particles to that of the brownian particle, for brevity taken here equal to 1 (see e.g. [25], ch.IX.4, [41] §5). We notice however, that (7.1) has been extrapolated far beyond the usual model of brownian motion, namely, a heavy particle interacting via hard collisions with a bath of much lighter particles (e.g. it has been used for self-gravitating systems, see the discussion in [43] $\S 5$ and references therein).

On the other hand, (7.1) is also obtained by using Langevin's equation as a dynamical model and assuming, that the motion of the particle is a Markov process, and that motion along $x$ is deterministic so that the dependence of the transition probability on $x$ is a $\delta$ function (see e.g. 24] eq(242), [25] ch.II.2, [40 §VIII.7). This is an extra condition, the use of which is reflected in the derivation of (7.1) in the context of StC by writing Langevin's equation as a stochastic differential equation

$$
\mathrm{d} x=y \mathrm{~d} t \quad \mathrm{~d} y=-(\beta y-F(x)) \mathrm{d} t+h \mathrm{~d} W_{t}
$$

where $W_{t}$ is a standard Wiener process and no stochastic term appears in $\mathrm{d} x$ (see e.g. [47], theorem 9.3.1, [39], §5.3.6, [42] §II.3). This extra condition is often justified by saying that the external field varies slowly on a length scale in which the velocity is damped (e.g. [40], p.232). Nevertheless, there is no generally accepted view on how (7.1) should be generalized if this extra condition is relaxed, although second order derivatives in $x$ are expected to appear on the basis of a systematic analysis of the microscopic dynamics of realistic classical 3Dmodels of brownian particles weakly coupled to the bath (44 §2, 455 §4). f. Such 2nd order derivatives are not easily accounted for by approaches based on StC, since this presupposes the appearance of a stochastic term in the first of eqs( 7.2$)$, not easily justified from a physical point of view.

On the other hand, in 13 $\S 5$ we have shown how such evolution equations (including (7.1) are formally incorporated as hamiltonian equations in symplectic mechanics developed in the context of the noncommutative DC defined by(5.21). Below we explore the physical interpretation of this formal result by employing the techniques and conceptual framework of the previous sections.

In the notation of $\S 5$ we write for a $(2+1)$ D-system

$$
\left(u^{\mu}\right)=(u, v, w), \quad\left(x^{\mu}\right)=(t, x, y), \quad\left(a_{i}\right)=(a, \bar{a}), \quad P^{\mu}=(p, q, r), \quad X=\sum_{\mu} P^{\mu} \partial_{+u^{\mu}}
$$

\footnotetext{
${ }^{6}$ The same conclusion follows from a similar analysis of quantum systems and by taking the classical limit of the resulting equations; e.g. for a harmonic oscillator in a bath of oscillators, such an analysis leads to a widely used kinetic equation of the Lindblad type (i.e. conserving density matrices), whose classical limit is a FP equation involving a drift and a diffusion term in the $x$ axis $(46$ § 3,4$)$.
} 


$$
\left(A_{\nu}^{\mu}\right)=\left(\begin{array}{ccc}
1 & 1 & 1 \\
\kappa & \lambda & \mu \\
\kappa^{\prime} & \lambda^{\prime} & \mu^{\prime}
\end{array}\right)
$$

Then, by (5.11), (5.15), (5.19), we have

$$
\begin{gathered}
X(x)=\langle\mathrm{d} x, X\rangle=a(\kappa p+\lambda q+\mu r)=\frac{a^{2}}{h_{11}} R_{x} \\
X(y)=\langle\mathrm{d} y, X\rangle=\bar{a}\left(\kappa^{\prime} p+\lambda^{\prime} q+\mu^{\prime} r\right)=\frac{\bar{a}^{2}}{h_{22}} R_{y} \\
\eta^{11}=\frac{a^{2}}{b}\left(\kappa^{2} p+\lambda^{2} q+\mu^{2} r\right), \quad \eta^{12}=\frac{a \bar{a}}{b}\left(\kappa \kappa^{\prime} p+\lambda \lambda^{\prime} q+\mu \mu^{\prime} r\right) \\
\eta^{22}=\frac{\bar{a}^{2}}{b}\left(\kappa^{\prime 2} p+\lambda^{\prime 2} q+\mu^{\prime 2} r\right)
\end{gathered}
$$

where the limiting probabilities in the continuous limit (5.3) are readily found via (7.4), (5.16) to be

$$
(\hat{p}, \hat{q}, \hat{r})=\left(\frac{\hat{\lambda} \hat{\mu}^{\prime}-\hat{\lambda}^{\prime} \hat{\mu}}{|\hat{A}|}, \frac{\hat{\kappa}^{\prime} \hat{\mu}-\hat{\kappa} \hat{\mu^{\prime}}}{|\hat{A}|}, \frac{\hat{\lambda^{\prime}} \hat{\kappa}-\hat{\lambda} \hat{\kappa}^{\prime}}{|\hat{A}|}\right), \quad|\hat{A}|=\operatorname{det}\left(\hat{A}_{\nu}^{\mu}\right)
$$

Finally, the evolution equation 5.18$)$ is

$$
\partial_{t} f-\frac{a^{2}}{b h_{11}} R_{x} \partial_{x} f-\frac{\bar{a}^{2}}{b h_{22}} R_{y} \partial_{x} f-\frac{1}{2}\left(\eta_{11} \partial_{x}^{2} f+\eta_{12} \partial_{x y}^{2} f+\eta_{22} \partial_{y}^{2} f\right)+o(a, \bar{a})=0
$$

Now suppose that $(x, y)$ are the phase space coordinates of a particle, moving under friction linear in the velocity $y$, say $-\beta y$, and an external field $F(x)$. Evidently, the generator of newtonian motion is $(y,-(\beta y-F(x))$ and therefore as in $\S 5$ (cf. (5.13)) we may introduce the 1 -forms

$$
\alpha_{1}=\mathrm{d} x+y \mathrm{~d} t, \quad \alpha_{2}=\mathrm{d} y-(\beta y-F(x)) \mathrm{d} t
$$

Consequently (5.14), giving the "infinitesimal" changes of $(\mathrm{d} x, \mathrm{~d} y)$ in time $\mathrm{d} t$, becomes $\left\langle\alpha_{i}, X\right\rangle=0, i=1,2$, hence

$$
\langle\mathrm{d} x, X\rangle=b y=b R_{x}, \quad\langle\mathrm{~d} y, X\rangle=-b(\beta y-F(x))=b R_{y}
$$

and in the limit

$$
R_{x}=\hat{R}_{x}=y, \quad R_{y}=\hat{R}_{y}=-(\beta y-F(x))
$$

If motion along $x$ is deterministic in the continuous limit, that is, the concept of a trajectory in $x$ is meaningful, then by the remark (3) in $\S 5$, this is equivalent to

$$
\hat{\eta}_{11}=0 \quad\left(\text { hence } \hat{\eta}_{12}=0\right), \quad \text { that is } \quad \hat{\kappa}^{2} \hat{p}+\hat{\lambda}^{2} \hat{q}+\hat{\mu}^{2} \hat{r}=0
$$


This corresponds to the absence of a stochastic term in (7.2i). Since $\hat{p}, \hat{q}, \hat{r}$ are nonnegative, it is readily obtained from $(7.13),(7.9)$ that up to a permutation of the lattice coordinates $(u, v, w)$ (hence of $(p, q, r))$, there are two possibilities:

(1) $\hat{q}=\hat{r}=0, \hat{p}=1, \hat{\kappa}=\hat{\kappa}^{\prime}=0$ implying $\hat{\eta}_{22}=0, \hat{\lambda} \hat{\mu}^{\prime}-\hat{\lambda}^{\prime} \hat{\mu} \neq 0$. Then, (5.20), the evolution equation in the continuous limit, is

$$
\partial_{t} f-y \partial_{x} f+(\beta y-F(x)) \partial_{y} f=0
$$

This describes motion with velocity damping and well defined trajectories in phase space. There is a 4-parameter gauge freedom for the transformation (7.4).

(2)

$$
\hat{q}=0, \quad \hat{p}=\frac{\hat{\mu}^{\prime}}{\hat{\mu}^{\prime}-\hat{\kappa}^{\prime}}, \quad \hat{r}=\frac{\hat{\kappa}^{\prime}}{\hat{\kappa}^{\prime}-\hat{\mu}^{\prime}}, \quad \kappa=\mu=0, \quad \hat{\eta}_{22}=-\hat{\kappa}^{\prime} \hat{\mu}^{\prime}>0
$$

with the evolution equation (5.20) being

$$
\partial_{t} f-y \partial_{x} f+(\beta y-F(x)) \partial_{y} f+\frac{1}{2}\left(h_{22} \hat{\kappa}^{\prime} \hat{\mu}^{\prime}\right) \partial_{y}^{2} f=0
$$

and there is again a 4-parameter gauge freedom subject to the constraint $\hat{\kappa}^{\prime} \hat{\mu}^{\prime}<0$. This is formally identical with the adjoint of (7.1). This formal similarity is more than a coincidence and gives another illustration of the consistency of the probabilistic framework introduced in $\S 3$ and elaborated in $\S 5$. A simple choice of the gauge is $\kappa^{\prime}=-\mu^{\prime}=1, \lambda^{\prime}=0$. Then, in view of (7.4), (5.16), eqs (5.19), (5.23) imply

$$
\eta^{22}=\frac{\bar{a}^{2}}{b}(p+r)+o(a, \bar{a}), \quad \frac{1}{b}\langle\mathrm{~d} y \bullet \mathrm{d} y, X\rangle=\eta^{22}+o(a, \bar{a})
$$

Therefore

$$
\frac{1}{b}\langle\mathrm{~d} y \bullet \mathrm{d} y, X\rangle=\frac{\bar{a}^{2}}{b}(p+r)+o(a, \bar{a})=\frac{\bar{a}^{2}}{b}(1-q)+o(a, \bar{a})=\frac{\bar{a}^{2}}{b}+o(a, \bar{a}, q) \rightarrow \hat{\eta}^{22}=h_{22}
$$

i.e. $\bar{a}^{2} / b$ is the average of the square of the velocity change from $y$ to $y+\mathrm{d} y$ in a "small" time interval $b$ divided by $b$.

On the other hand, it is well known that in the usual interpretation of (7.1) as a model of brownian motion, $h=\beta \bar{y}^{2}$, with $\bar{y}=$ thermal velocity, whereas, for short times $t$ in which the velocity changes from $y_{0}$ to $y,\left\langle\left(y-y_{0}\right)^{2}\right\rangle / t=\beta \bar{y}^{2}+o(t)=h+o(t)$ with $\langle$,$\rangle denoting$ the average over the bath (see e.g. [24] eq(161)). Thus, in the continuous limit $h_{22} \equiv h$ and (7.16) coincides with the adjoint of (7.1).

It should be noticed here, that this result follows by assuming that in the configuration space (i.e. in the $x$-axis), motion is along well-defined trajectories. It is a remarkable fact however, that if this assumption is relaxed, then (7.10) reduces to an equation describing diffusion in the $x$-axis as well, a fact not easily accommodated in other approaches, as explained at the beginning of this section.

Remarks: (a) In the present section and in $\S 4$, we have seen that up to a coordinate transformation, our approach allows for concrete results in specific cases, once the drift term 
$\left\langle\mathrm{d} x^{i}, X\right\rangle$ i.e. the average "infinitesimal" change of $x^{i}$ is given, that is, once a prescription is given for the choice of $X$ in each particular case. In [13], $X$ was specified by assuming it to be hamiltonian in the context of the "second order calculus" (5.21). This points out to the need of developing symplectic mechanics on the oriented hypercubic lattice, to be presented in another paper.

(b) A similar, but simpler procedure yields in the 1D case the following: We write (5.1), (5.3) as

$$
t=-b(u+v), \quad x=a(\kappa u+\lambda v), \quad \frac{a^{2}}{b} \longrightarrow h
$$

Then using (4.1), eq(5.16) becomes

$$
\hat{p}=-\frac{\hat{\lambda}}{\hat{\kappa}-\hat{\lambda}}, \quad \hat{p}=\frac{\hat{\kappa}}{\hat{\kappa}-\hat{\lambda}}, \quad \hat{\lambda} \hat{\kappa}<0
$$

and the evolution equation in the continuous limit, eq(5.20), gives

$$
\partial_{t} f-\hat{R} \partial_{x} f+\frac{1}{2} h(\hat{\lambda} \hat{\kappa}) \partial_{x}^{2} f=0
$$

Notice that $\langle\mathrm{d} x, X\rangle / b=a(\kappa p+\lambda q) / b=a^{2} R / b h \longrightarrow \hat{R}$ so that the results of $\S 4$ are recovered if $\hat{\kappa}=-\hat{\lambda}=1$ (cf. eqs(4.6)-(4.10) ).

\section{Comments on higher order equations}

The basic methodological "rules" of the present approach introduced in the previous sections are:

- The extended phase-space has the structure of a (oriented hypercubic) lattice $\mathcal{M}$.

- The phase space coordinates $\left(x^{\mu}\right)$ are obtained by a coordinate transformation from the lattice coordinates $\left(u^{\mu}\right)$, involving the scaling parameters $\left(a_{\mu}\right)$, so that the usual continuum description in terms of the $\left(x^{\mu}\right)$ is recovered in the limit (5.3) (cf. [32], p.244).

In principle however, one can imagine other limits as well. In this section we first examine this possibility in a somewhat more general setting and then we reconsider the case of the oriented hypercubic lattice in the light of the results obtained.

Let the $\mathrm{DC}$ on $\mathcal{M}$ be defined by

$$
\mathrm{d} u^{\mu} \bullet \mathrm{d} u^{\nu}=C_{\rho}^{\mu \nu} \mathrm{d} u^{\rho}, \quad C_{\rho}^{\mu \nu} \in \mathcal{A}
$$

where the summation convention has been used (it is used throughout this section). This may be considered as a class of (algebraic) deformations of the ordinary DC. It can be shown that $([20] \S 4.5)$

$$
\mathrm{d} f=D_{\rho} f \mathrm{~d} u^{\rho}, \quad f=f(u) \in \mathcal{A}
$$




$$
D_{\rho} f=\partial_{\rho} f+\sum_{r=2}^{+\infty} \frac{1}{r !} \sum_{\substack{\mu_{1}, \ldots, \mu_{r} \\ \nu_{1}, \ldots, \nu_{r}-2}} C_{\nu_{1}}^{\mu_{1} \mu_{2}} C_{\nu_{2}}^{\mu_{3} \nu_{1}} \cdots C_{\rho}^{\mu_{r} \nu_{r-2}} \partial_{\mu_{1}} \cdots \partial_{\mu_{r}} f
$$

We pass to the continuous limit by dividing $\left\{u^{\mu}\right\}$ into several groups, with the same scaling in each group. In each case one of the groups contains the "time" variable(s). It will be readily seen, that the results obtained are valid even if in each group, each coordinate is scaled differently, provided the order of magnitude is the same for all elements of the same group. Moreover, any finite limit of the scaling parameters is set equal to 1 , to avoid useless cumbersome notation.

(1) Division of $\left\{u^{\mu}\right\}$ into two groups:

$$
u^{a}=: \frac{t^{a}}{b}, \quad a=0, \ldots, n, \quad u^{i}=: \frac{x^{i}}{a}, \quad i=n+1, \ldots, N
$$

continuous limit:

$$
a, b \rightarrow 0, \quad \frac{a^{2}}{b} \rightarrow 1
$$

From $(8.1)$ and $(8.2)$ we get

$$
\begin{gathered}
\mathrm{d} t^{a} \bullet \mathrm{d} t^{b}=b C_{c}^{a b} \mathrm{~d} t^{c}+\frac{b^{2}}{a} C_{i}^{a b} \mathrm{~d} x^{i} \\
\mathrm{~d} t^{a} \bullet \mathrm{d} x^{i}=a C_{b}^{a i} \mathrm{~d} t^{b}+b C_{j}^{a i} \mathrm{~d} x^{j} \\
\mathrm{~d} x^{i} \bullet \mathrm{d} x^{j}=\frac{a^{2}}{b} C_{a}^{i j} \mathrm{~d} t^{a}+a C_{k}^{i j} \mathrm{~d} x^{k} \\
D_{a} f=\partial_{a} f+\frac{1}{2} b C_{a}^{b c} \partial_{b} \partial_{c} f+a C_{a}^{b i} \partial_{b} \partial_{i} f+\frac{1}{2} \frac{a^{2}}{b} C_{a}^{i j} \partial_{i} \partial_{j} f+o(a) \\
D_{i} f=\partial_{i} f+\frac{1}{2} \frac{b^{2}}{a} C_{i}^{a b} \partial_{a} \partial_{b} f+b C_{i}^{a j} \partial_{a} \partial_{j} f+\frac{1}{2} a C_{i}^{j k} \partial_{j} \partial_{k} f+o(a)
\end{gathered}
$$

If as in $\S 5$, quantities with a hat denote values in the limit (8.3), then in that limit, (8.4), (8.5) reduce to (cf. eqs(5.8), (5.21) and [13] eq.(4.13))

$$
\begin{array}{rc}
\mathrm{d} t^{a} \bullet \mathrm{d} t^{b}=0, \quad \mathrm{~d} t^{a} \bullet \mathrm{d} x^{i}=0, \quad \mathrm{~d} x^{i} \bullet \mathrm{d} x^{j}=\hat{C}_{a}^{i j} \mathrm{~d} t^{a} \\
D_{a} f=\partial_{a} f+\frac{1}{2} \hat{C}_{a}^{i j} \partial_{i} \partial_{j} f, \quad D_{i} f=\partial_{i} f
\end{array}
$$

so that the evolution equation (3.34) reduces to a well-defined equation of the form (5.20).

\section{(2) Division of $\left\{u^{\mu}\right\}$ into three groups:}

$u^{a}=: \frac{t^{a}}{b}, a=0, \ldots, m, \quad u^{r}=: \frac{y^{r}}{c}, r=m+1, \ldots, n, \quad u^{i}=: \frac{x^{i}}{a}, i=n+1, \ldots, N$ 
continuous limit:

$$
a, b, c \rightarrow 0, \quad \frac{a^{2}}{c} \rightarrow 1, \quad \frac{a^{3}}{b} \rightarrow 1
$$

Eqs (8.1), (8.2) imply

$$
\begin{aligned}
& \mathrm{d} t^{a} \bullet \mathrm{d} t^{b}=b C_{c}^{a b} \mathrm{~d} t^{c}+\frac{b^{2}}{c} C_{r}^{a b} \mathrm{~d} y^{r}+\frac{b^{2}}{a} C_{i}^{a b} \mathrm{~d} x^{i} \\
& \mathrm{~d} t^{a} \bullet \mathrm{d} y^{r}=c C_{b}^{a r} \mathrm{~d} t^{b}+b C_{s}^{a r} \mathrm{~d} y^{s}+\frac{b c}{a} C_{i}^{a r} \mathrm{~d} x^{i} \\
& \mathrm{~d} t^{a} \bullet \mathrm{d} x^{i}=a C_{b}^{a i} \mathrm{~d} t^{b}+\frac{a b}{c} C_{r}^{a i} \mathrm{~d} y^{r}+b C_{j}^{a i} \mathrm{~d} x^{j} \\
& \mathrm{~d} y^{r} \bullet \mathrm{d} y^{s}=\frac{c^{2}}{b} C_{a}^{r s} \mathrm{~d} t^{a}+c C_{t}^{r s} \mathrm{~d} y^{t}+\frac{c^{2}}{a} C_{i}^{r s} \mathrm{~d} x^{i} \\
& \mathrm{~d} y^{r} \bullet \mathrm{d} x^{i}=\frac{a c}{b} C_{a}^{r i} \mathrm{~d} t^{a}+a C_{s}^{r i} \mathrm{~d} y^{s}+c C_{j}^{r i} \mathrm{~d} x^{j} \\
& \mathrm{~d} x^{i} \bullet \mathrm{d} x^{j}=\frac{a^{2}}{b} C_{a}^{i j} \mathrm{~d} t^{a}+\frac{a^{2}}{c} C_{r}^{i j} \mathrm{~d} y^{r}+a C_{k}^{i j} \mathrm{~d} x^{k} \\
& D_{i} f=\partial_{i} f+o(a), \frac{D_{r} f=\partial_{r} f+\frac{1}{2} \frac{a^{2}}{c} C_{r}^{i j} \partial_{i} \partial_{j} f+o\left(a^{3}\right)}{D_{a} f=\partial_{a} f+\frac{1}{2} \frac{a^{2}}{b} C_{a}^{i j} \partial_{i} \partial_{j} f+\frac{c a}{b} C_{a}^{r i} \partial_{r} \partial_{i} f+} \\
& \frac{1}{6}\left(C_{a}^{i l} C_{l}^{j k}+C_{a}^{i r} C_{r}^{j k}+C_{a}^{i b} C_{b}^{j k}\right) \partial_{i} \partial_{j} \partial_{k} f+o(a)
\end{aligned}
$$

It is now clear that in the limit (8.8), eqs 8.10 ) and all but the last one of (8.9) have a well-defined limit. The coefficient of $C_{a}^{i j}$ in the last of (8.9) and in (8.11) diverges as 1/a. Therefore, the limit (8.8) exists in this case only if we impose an extra condition on the structure constants which define the DC

$$
C_{a}^{i j}=a K_{a}^{i j}, \quad K_{a}^{i j} \rightarrow \hat{K}_{a}^{i j}
$$

In this case (8.9)-8.11) reduce in the continuous limit (8.8):

$$
\begin{gathered}
\mathrm{d} t^{a} \bullet \mathrm{d} t^{b}=0, \quad \mathrm{~d} t^{a} \bullet \mathrm{d} y^{r}=0, \quad \mathrm{~d} t^{a} \bullet \mathrm{d} x^{i}=0, \quad \mathrm{~d} y^{r} \bullet \mathrm{d} y^{s}=0, \\
\mathrm{~d} y^{r} \bullet \mathrm{d} x^{i}=\hat{C}_{a}^{r i} \mathrm{~d} t^{a}, \quad \mathrm{~d} x^{i} \bullet \mathrm{d} x^{j}=\hat{K}_{a}^{i j} \mathrm{~d} t^{a}+\hat{C}_{r}^{i j} \mathrm{~d} y^{r} \\
D_{i} f=\partial_{i} f, \quad D_{r} f=\partial_{r} f+\frac{1}{2} \hat{C}_{r}^{i j} \partial_{i} \partial_{j} f \\
D_{a} f=\partial_{a} f+\hat{C}_{a}^{r i} \partial_{r} \partial_{i} f+\frac{1}{2} \hat{K}_{a}^{i j} \partial_{i} \partial_{j} f+\frac{1}{6} \hat{C}_{a}^{i r} \hat{C}_{r}^{j k} \partial_{i} \partial_{j} \partial_{k} f
\end{gathered}
$$

so that the evolution equation (3.34) reduces to an equation containing 3rd order derivatives. 
It is easily seen that proceeding in this way, that is, by dividing $\left\{u^{\mu}\right\}$ into disjoint subsets, scaled by parameter $a_{A}, A=1,2, \ldots, L$ and then considering the limits

$$
a_{A} \rightarrow 0, \quad \frac{\left(a_{1}\right)^{A}}{a_{A}} \rightarrow \text { finite, } \quad C_{\rho}^{\mu \nu} \rightarrow \text { finite }
$$

then unless $L=2$, extra conditions on $C_{\rho}^{\mu \nu}$ should be imposed so that in that limit the commutation relations and $\mathrm{d} f, f \in \mathcal{A}$, are well-defined.

The significance of this general result can be better appreciated if we implement the procedure described above, in the case of the oriented hypercubic lattice $\mathcal{M}$, that is, when the DC is that of $\S 5,(5.8)$. Instead of the limit (5.3) (or equivalently (8.3)), we consider

$$
a_{\mu} \rightarrow 0, \quad \frac{a_{i} a_{j} a_{k}}{b} \rightarrow h_{i j k}, \quad \frac{a_{i}}{b} \rightarrow \pm \infty, \quad A_{\nu}^{\mu} \rightarrow \hat{A}_{\nu}^{\mu}
$$

that is, essentially the limit (8.8) (to simplify the presentation, no coordinates of order $b^{2 / 3}$ are considered since they lead to the results of $\$ 5$ ). However, it will become evident that the results obtained are valid for any limit of the type (8.15).

By (8.16) we have

$$
a_{i}=\bar{b} \tilde{a}_{i}, \quad \tilde{a}_{i} \tilde{a}_{j} \tilde{a}_{k} \rightarrow h_{i j k}, \quad \bar{b}:=b^{1 / 3}
$$

For arbitrary $f \in \mathcal{A}, \mathrm{d} f$ is given by (5.5) and we consider its value in the limit (8.16): Expanding (5.6) and using that $\sum_{\mu} B_{i}^{\mu}=\sum_{\mu} A_{\mu}^{0} B_{i}^{\mu}=0$, once again we get (8.10i), namely

$$
\bar{\partial}_{i} f=\partial_{i} f+o(\bar{b})
$$

Similarly, (5.7) gives

$$
\begin{aligned}
\frac{1}{2} \Delta f & =\frac{1}{2 \bar{b}} \sum_{j, k}\left(\sum_{\mu}\left(\tilde{a}_{j} A_{\mu}^{j}\right)\left(\tilde{a}_{k} A_{\mu}^{k}\right) B_{0}^{\mu}\right) \partial_{j} \partial_{k} f \\
& +\frac{1}{6} \sum_{j, k, l}\left(\sum_{\mu}\left(\tilde{a}_{j} A_{\mu}^{j}\right)\left(\tilde{a}_{k} A_{\mu}^{k}\right)\left(\tilde{a}_{l} A_{\mu}^{l}\right) B_{0}^{\mu}\right) \partial_{j} \partial_{k} \partial_{l} f+o(\bar{b})
\end{aligned}
$$

To simplify the notation, we put

$$
A_{\mu}(\xi):=\sum_{j} \tilde{a}_{j} A_{\mu}^{j} \xi_{j} \rightarrow \hat{A}_{\mu}(\xi), \quad \xi:=\left(\xi_{j}\right) \in \mathbb{R}^{N}
$$

and we consider the functions

$$
\vartheta_{2}(\xi)=\frac{1}{2 \bar{b}} \sum_{\mu}\left(A_{\mu}(\xi)\right)^{2} B_{0}^{\mu}, \quad \vartheta_{3}(\xi)=\frac{1}{6} \sum_{\mu}\left(A_{\mu}(\xi)\right)^{3} B_{0}^{\mu},
$$

related to the coefficients in (8.18). We notice that by the Schwartz inequality

$$
\left|A_{\mu}(\xi)\right| \leq\|\xi\|\left\|A_{\mu}\right\| \leq\|\xi\| \max _{\mu}\left\{\left\|A_{\mu}\right\|\right\}=: M\|\xi\| \rightarrow \hat{M}\|\xi\|
$$


where $A_{\mu}=\left(\tilde{a}_{j} A_{\mu}^{j}\right) \in \mathbb{R}^{N}$ and \|\| is the Euclidean norm in $\mathbb{R}^{N}$. Therefore,

$$
\left|\vartheta_{3}(\xi)\right| \leq \frac{M\|\xi\|}{6} \sum_{\mu}\left(A_{\mu}(\xi)\right)^{2}\left|B_{0}^{\mu}\right| \rightarrow \frac{\hat{M}\|\xi\|}{6} \sum_{\mu}\left(\hat{A}_{\mu}(\xi)\right)^{2} \hat{B}_{0}^{\mu}
$$

where we used that $B_{0}^{\mu} \rightarrow \hat{B}_{0}^{\mu} \geq 0$, eq (5.17).

On the other hand, if we require that (8.18) is well defined in the limit (8.17), then $\sum_{\mu}\left(A_{\mu}(\xi)\right)^{2} B_{0}^{\mu}$ in $8.19 \mathrm{i}$ ) should be at least of order $\bar{b}$ (cf. (8.12)) and therefore, its limiting value should be zero, i.e.

$$
\sum_{\mu}\left(\hat{A}_{\mu}(\xi)\right)^{2} \hat{B}_{0}^{\mu}=0
$$

Therefore, $\vartheta_{3}(\xi)=0$ for all $\xi \in \mathbb{R}^{N}$, that is, the coefficients of 3rd derivatives of $f$ in (8.18) vanish.

We remark the following:

(i) From the form of (8.18), (8.19), it is clear that if we require $\mathrm{d} f$ to be well-defined in the limit (8.17), then all coefficients of the derivatives of order higher than the second in the expansion of $\mathrm{d} f$, eq(5.5), vanish.

(ii) The same conclusion holds if we consider the limit (8.15), that is, instead of (8.17), $a_{i}=\bar{b}^{1 / L} \tilde{a}_{i}, L \geq 3$.

(iii) For the above result, it is essential that $\hat{B}_{0}^{\mu} \geq 0$. This is a consequence of our interpretation of vector fields as transition probability distributions. In fact, the procedure followed above, is a modification of an argument used in the theory of Markov processes which ensures that higher than the second moments of such processes vanish ([39], §3.4).

(iv) It can be seen from (8.18), that in the limit (8.17), the resulting second order coefficients are no longer nonnegative-definite (see also (8.14)).

Therefore, summarizing our results in this section, we may say that a continuous description can be obtained from any discrete lattice structure only if the discrete phase space coordinates are at least of order $\sqrt{t}$, where $t$ is the time. In this case we get evolution equations at most of the 2 nd order with nonnegative-definite 2 nd order coefficient, which is the most general (linear autonomous) differential generator conserving probabilities ([30 $\S \S 4,5)$.

\section{Discussion}

In this section we summarize the basic assumptions introduced in this paper, results and conceptual insights obtained and we comment on the limitations of the present approach and on directions in which it can be further elaborated.

We started with a discrete picture of the extended phase space $\mathcal{M}$. This corresponds to looking at all fine structural details of the system and subsequently pass to its "coarser" description, which corresponds to the limiting procedures followed in $\S \S 4-8$, as for instance done in statistical mechanics and lattice field theory.

On the other hand, we saw in $\S 3$ that a discrete picture allows for a geometric visualization of the universal DC on $\mathcal{M}$ as a di-graph, from which special DC on $\mathcal{M}$ can be easily 
constructed. In this way, their necessarily noncommutative character discussed in $\S 3.1$ is interpreted as due to the non-vanishing "size" of the differentials. In fact, these general ideas were illustrated in $\S 2$. The $1 \mathrm{D}$ discrete model studied there, clearly suggested connections between motion defined on $\mathcal{M}$ endowed with a noncommutative $\mathrm{DC}$, and a random walk on an oriented square lattice, one dimension of which is related to time (cf. (2.4)). In $\S 3$, vector fields $X$ were defined as elements of the space dual to 1-forms and turn to be 1st order difference operators on the algebra $\mathcal{A}$ of functions on $\mathcal{M}$, when $\mathcal{M}$ is an $(N+1)$-dimensional oriented hypercubic lattice. Here, a crucial fact is that only vector fields of a special form are the generators of automorphisms of $\mathcal{A}$, in contrast to the usual (commutative) DC, where any vector field $X$ (i.e. 1st order differential operator) generates (local) automorphisms of $\mathcal{A}$. That a vector field generates automorphisms of $\mathcal{A}$ is equivalent to the fact that it induces a flow of well defined trajectories on $\mathcal{M}$. In the discrete case, these are paths which connect points of the lattice. This raises the question of what kind of motion is described by an arbitrary vector field Thus, we have been led to a new way of looking at vector fields, namely, both as generators of evolution of observables and as states describing transition probabilities for "infinitesimal" changes on the lattice.

In this new perspective, probabilistic concepts are introduced in the dynamics at the "infinitesimal level", in contrast to (classical) statistical mechanics, where microscopic dynamics and probability distributions are two a priori quite distinct concepts. Mathematically speaking, this double role of vector fields stems from the fact that in the present context, the concept of a vector field is much more general than the generator of an automorphism of $\mathcal{A}$, as in the case of classical dynamics based on ordinary DC (cf. the discussion at the end of $\S 3.2$ ). In fact, this is a general feature of noncommutative DC on both discrete and "continuous" manifolds, which allows for the description of evolution equations involving higher than the 1st derivatives and/or difference operators: By using the concept of a vector field, a noncommutative version of differential geometry and tensor calculus can be developed in close analogy with the ordinary (commutative) case. This is of potential value in many areas of physics (see e.g. [20], [12], [13]). We will come back to this point at the end of this section.

An indication for the consistency of the interpretation of vector fields $X$ as giving the transition probability distribution for "infinitesimal" motion along the lattice axes, is provided by the proof that the associated correlation matrix $\mathbb{P}=\left(P^{\mu \nu}\right)$ for the differentials of the lattice coordinates, vanishes if and only if $X$ generates automorphisms of $\mathcal{A}$, or equivalently, if trajectories along the lattice are well defined. This means that there is no possible interference of motions along different axes at the same point (motion along a specific axis is either impossible, or certain). Moreover, in this way it became clear that, a function $f$ on $\mathcal{M}$ is an observable, in the sense that expectation values for its change represented by $\mathrm{d} f$, are given by $\langle\mathrm{d} f, X\rangle$, multiplication of observables corresponding to the $\bullet$-product in $\Omega^{1}$, eq(1.3). On the basis of this and assuming on physical grounds that time flows with certainty, i.e. for every vector field $X$ there is always a change of some fixed element, $t$ say, of $\mathcal{A}$, it follows that $\mathrm{d} t$ is proportional to the (essentially) unique eigenvector of the above mentioned correlation matrix $\left(P^{\mu \nu}\right)$ belonging to the zero eigenvalue. It turns out to be the unit $\rho$ of the commutative algebra $\left(\Omega^{1}(\mathcal{A}), \bullet\right)$ of 1 -forms, $\mathrm{d} t=-b \rho(\S \S 3.3,3.4)$. Using these results, the generator of dynamical evolution, $X$, is determined by $N 1$-forms $\alpha^{i}$ vanishing along $X$, eq(5.14) and the time evolution of observables is given by $-X(f) / b=0$, eq( 3.34$)$, in direct analogy with classical dynamics. 
In $\S \S 4,5$, using the probabilistic framework of $\S 3$, we considered appropriately scaled linear coordinate transformations on the lattice. We passed to the continuous limit in which $\mathcal{M}$ becomes $\mathbb{R}^{N+1}$ in the same way this is done in the theory of brownian motion. We showed that the evolution equation for observables is a 2nd order partial differential equation with nonnegative-definite leading coefficient. This is the limit of the correlation matrix $\left(P^{\mu \nu}\right)$ of (changes along) the lattice axes, namely $\left(\hat{\eta}^{i j}\right)$ in (5.19) or (5.25). Each of its elements measures the deviation of the evolution operator from an ordinary derivation (i.e. generator of deterministic motion) in the corresponding phase space axis. Thus, noncommutativity of the DC in the continuous limit (that is, in a "coarse-grained" picture of the system's evolution) is due to the fact that on a discrete ("fine") level, motion described by a vector field, is in general not along well defined phase-space trajectories. This is an idea also appearing in the theory of brownian motion and stochastic mechanics, though in a completely different mathematical and conceptual framework (see e.g. [48], 449, 442). In fact, the explicit form of $\left(\hat{\eta}^{i j}\right)$ in (5.25) (cf. (5.23) as well) is consistent with the form of the diffusion matrix in the kinetic theory of open systems, the theory of markovian stochastic processes and StC. Moreover, the coefficients of 1 st order derivatives in the evolution equation (5.20), are just the first moments of the coordinate changes with respect to the transition probability distribution defined by the vector field $X$ (cf. (5.11), (5.15)). Therefore, our evolution equation (5.20) is the formal adjoint of a generalized FP equation (backward equation), justifying our interpretation of $f \in \mathcal{A}$ as observables and thus giving still another indication of the consistency of our approach. An interesting conclusion in this context is that the interpretation of vector fields as transition probability distributions on the lattice, implies in the continuous limit that evolution is forward in time. Thus, if we accept the correspondence

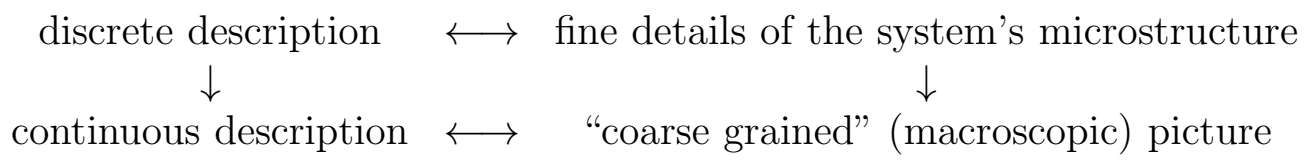

then, in the present conceptual framework, irreversible evolution is a characteristic of macroscopic systems.

The continuous limit considered in $\S 5$, at first sight may appear an arbitrary choice among many possible ones that would lead (via the expansion of (5.10)) to evolution equations in general involving derivatives of any order. This issue was studied in $\S 8$ and we have shown that (i) the limiting procedure of $\S 5$ is the only way to pass to a continuous description by scaling the lattice coordinates, without imposing additional ad hoc conditions on the DC, that is, it is the only continuous limit independent of the general form of the DC one starts with; (ii) if the probabilistic interpretation of vector fields introduced in $\S 3$ is employed, then for all types of limiting procedures, higher than the second order differential equations are impossible as evolution equations. Thus, (i) and (ii) imply the unique character of the limit considered in $\S 5$.

In $\S \S 4,7$ we considered simple applications of our approach:

(a) The derivation of the 1D diffusion and Smoluchowski equations for a constant external field, and the 1D FP equation in velocity space giving the Ornstein-Uhlenbeck process, thus illustrating the possibility to incorporate random walk models in the present context.

(b) The derivation of Kramers' equation in one spatial dimension, by assuming (i) (newtonian) motion under friction linear in the velocity and an external field; (ii) trajectories 
exist in configuration space, that is, motion is deterministic there. In fact, by using the explicit form of the correlation matrix $\hat{\eta}^{i j}$ under the above assumptions, we have shown that the diffusion coefficient in our evolution equation coincides with the diffusion coefficient of Kramers' equation computed in the context of the theory of brownian motion, or of kinetic theory.

On the other hand, our result makes explicit the use of assumption (ii) above, a fact often hidden in the derivations of Kramers' equation from physically plausible stochastic models. Although this is also made explicit when Kramers' equation is derived from Langevin's equation seen as a stochastic differential equation, in our opinion, the present approach has some advantage: In the context of StC, it is not easy to relax this assumption (see the discussion following (7.2)). In the present context however, this is possible. Actually, the general form of the corresponding corrections is evident, namely, a diffusion term in the configuration space, much in accordance with what seems plausible on the basis of the (classical and quantum) kinetic theory of open systems. Finally, it is clear that these results are valid in the $3 \mathrm{D}$ case.

The approach elaborated in this paper, clarifies several conceptual issues concerning the relevance of noncommutative DC to (the derivation of) kinetic equations and to StC. In addition, by giving a definite prescription for the general form of irreversible evolution equations in terms of vector fields $X$ in the discrete framework, it provides an adequate discrete formalism for deriving such equations in an appropriate continuous limit. However, it should be supplemented by a "dynamics", that is, a general procedure for choosing the generator $X$ in particular cases. In sections $\S 5$ and 7, we have used Newton's equations written as 1-form relations (see (5.13) and the derivation of (5.15), (7.12)). As briefly discussed at the end of $\S 7$, a more systematic approach is to develop symplectic mechanics in the present context and require $X$ to be hamiltonian, in analogy with classical mechanics. In fact, from a mathematical point of view, this approach has been followed successfully in the continuous regime for the second order DC defined by (5.21), giving the promising result that hamiltonian evolution equations have the form of generalized FP equations that appear in kinetic theory ([13]). In another paper, the present approach will be elaborated in this direction.

It is also possible (and physically desirable) to consider the extension of the present formalism when the transformation from the lattice coordinates $\left(u^{\mu}\right)$ to the scaled coordinates $\left(t, x^{i}\right)$, eq(5.1), is not linear. Many of the present results are expected to be still valid.

Finally, the general formalism and the conceptual framework in this paper, may be extended from the case of the oriented hypercubic lattice, to more general structures induced by appropriate DC on a discrete manifold. More precisely, it is clear that throughout this work, differentials are not necessarily infinitesimal quantities in the usual geometric sense of ordinary DC. Rather than that, if seen in the suggestive representation of a DC as a di-graph, they express interrelations between points of the discrete manifold, so that all points related to a given one, should be considered as being neighboring to it (see (3.5), its interpretation in $\S 3.1$ and the differentials for the hypercubic lattice, eq(3.21)). This idea, that a DC is based on a concept of "relational", rather than geometric type of "proximity" may be further elaborated. In this way, it may become possible to develop a general mathematical and conceptual framework for describing physical systems based on the interrelations (interactions) among its different parts, rather than on their relative geometric position. This is virtually relevant in situations where collective effects are significant, or even dominant (e.g. systems 
with long-range interactions), for which traditional approaches often do not work beyond the lowest approximation. We will come back to this point in another work.

\section{Appendix A}

1. Here we prove the following proposition referred to in $\S 3.2$.

Proposition $A$ 1-1 and onto mapping $\Phi: \mathcal{M} \mapsto \mathcal{M}$ induces an automorphism $\phi: \mathcal{A} \mapsto \mathcal{A}$ that maps the basis $\left\{e_{i}, i \in \mathcal{M}\right\}$ of $\mathcal{A}$ onto itself. The converse is also true.

Proof: " $\Rightarrow "$ : We define

$$
\phi: \mathcal{A} \mapsto \mathcal{A}: \quad \phi(f)(i)=f(\Phi(i))
$$

Clearly $\phi$ is an endomorphism and

$$
\phi\left(e_{i}\right)(j)=e_{i}(\Phi(j))=\delta_{i, \Phi(j)}=\delta_{\Phi^{-1}(i), j}=e_{\Phi^{-1}(i)}(j)
$$

hence

$$
\phi\left(e_{i}\right)=e_{\Phi^{-1}(i)}
$$

which shows that $\phi$ is $1-1$ and onto.

" $\Leftarrow "$ : Conversely, let $\phi$ be an automorphism of $\mathcal{A}$. From $(3.2 \mathrm{j})$ we get $\phi\left(e_{i}\right)\left(\phi\left(e_{j}\right)-\delta_{i j}\right)=$ 0 . If $\phi\left(e_{i}\right)=\sum_{k} f_{i k} e_{k}$, the above equation implies that $f_{i k}=0$ or $1, \forall i, k$. By (3.2 $\mathrm{ii}$ ) $\sum_{i, k} f_{i k} e_{k}=\sum_{k} e_{k}$ hence $\sum_{i} f_{i k}=1$ where we have used that $\phi(1)=1$ and $\left\{e_{i}\right\}$ is linearly independent. Consequently, in each column $k$ of $f_{i k}$ there exists exactly one element, on the $i_{k}$-th row, say, which is nonzero and therefore, necessarily equal to 1 . Now suppose that for $k \neq l$, the corresponding nonzero elements are on the same $i$-th row. Then, these columns are identical which is impossible, since $\phi$ is 1-1 and onto. Therefore, in each row $i$ of $f_{i k}$, exactly one $k$ is nonzero and equal to 1 . Hence, $\phi\left(e_{i}\right)=e_{k}$ for some $k$ depending on $i$. Now, $\phi^{-1}\left(e_{i}\right)=e_{\Phi(i)}$ defines $\Phi: \mathcal{M} \longmapsto \mathcal{M}$ which is 1-1 and onto and satisfies (A.1).

2. By considering the case of classical dynamics, we explain below the choice of the sign in (3.33) and comment on the form of (5.14).

Let the equations of motion of an $N$-dimensional dynamical system be

$$
\frac{\mathrm{d} x^{i}}{\mathrm{~d} s}=R^{i}(x)
$$

The time evolution of observables $f$ and states $\sigma$, is given by semigroups of Koopman and Perron-Frobenius operators respectively, whose infinitesimal generators lead to

$$
\frac{\partial f}{\partial s}=R^{i}(x) \frac{\partial f}{\partial x^{i}}, \quad \frac{\partial \sigma}{\partial s}=-\frac{\partial}{\partial x^{i}}\left(R^{i}(x) \sigma\right)
$$

with the summation convention used here. The operators on the r.h.s. are formal adjoints to each other (cf. e.g. [23] ch.7). 
Then, the prescription for extended dynamics is

equations of motion:

$$
\begin{array}{ccc}
\frac{\mathrm{d} x^{i}}{\mathrm{~d} s}=R^{i}(x), & \frac{\mathrm{d} t}{\mathrm{~d} s}=1 \\
\left(\frac{\partial}{\partial t}+\frac{\partial}{\partial x^{i}} R^{i}(x) \cdot\right) \sigma & =: & X_{S}(\sigma)=0 \\
\left(-\frac{\partial}{\partial t}+R^{i}(x) \frac{\partial}{\partial x^{i}}\right) f & =: & X_{O}(f)=0
\end{array}
$$

evolution of observables:

Therefore, the system of characteristic equations of the evolution equation for observables, is given by the vector field with components $\left(X_{O}(t), X_{O}\left(x^{i}\right)\right)=\left(-1, R^{i}\right)$, i.e. evolution is obtained by integrating the equations of motion "backwards in time". This explains the $(-)$ sign in (3.33) and the form of (5.13). In fact, to the system of characteristic equations of the observables' evolution equation (A.3iii) are associated the 1-forms

$$
\alpha^{i}=\mathrm{d} x^{i}+R^{i} \mathrm{~d} t \quad i=1,2, \ldots, N
$$

vanishing along the integral curves of the vector field $X_{O}$. This is equivalent to

$$
\left\langle\alpha^{i}, X_{O}\right\rangle=0 \quad i=1,2, \ldots, N
$$

$\langle$,$\rangle denoting the contraction of 1$-forms with vector fields in the ordinary DC. In the conceptual framework of the present paper, this is given by (5.13), (5.14). It is a completely general formulation that includes as special cases (in an obvious notation)

Newton's equations $(N=2 M)$

$$
\beta^{r}=\mathrm{d} q^{r}+v^{r} \mathrm{~d} t, \quad \gamma^{r}=\mathrm{d} v^{r}+f^{r} \mathrm{~d} t
$$

or Hamilton's equations

$$
\beta^{r}=\mathrm{d} q^{r}+\frac{\partial H}{\partial p_{r}} \mathrm{~d} t, \quad \gamma_{r}=\mathrm{d} p_{r}-\frac{\partial H}{\partial q^{r}} \mathrm{~d} t
$$

To connect this with the usual formulation of symplectic mechanics, we notice that, by introducing the symplectic matrix

$$
\left(J^{i j}\right)=\left(J_{i j}\right)=\left(\begin{array}{cc}
0 & I \\
-I & 0
\end{array}\right)
$$

( $I$ being the $M \times M$ identity matrix), the 1 -forms above can be rewritten in a form identical to (5.13), namely

$$
\alpha^{i}=\mathrm{d} x^{i}+\sum_{j} J^{i j} \frac{\partial H}{\partial x^{j}} \mathrm{~d} t \quad \Longleftrightarrow \quad \alpha_{i}=\sum_{j} J_{i j} \mathrm{~d} x^{j}-\frac{\partial H}{\partial x^{i}} \mathrm{~d} t
$$

\footnotetext{
${ }^{7} \mathrm{cf}$. the theory of stochastic processes and stochastic differential equations (e.g. 447, §2.6), where, for observables (i.e. bounded functions of a stochastic process): Generator of the evolution of observables $=$ (backward generator of evolution of the probability distribution) $=-($ adjoint of forward generator of evolution of the probability distribution).
} 
$(i=1,2, \ldots, 2 M)$ so that the $\alpha_{i}$ can be given in terms of the symplectic form

$$
\omega:=\frac{1}{2} \sum_{i, j} J_{i j} \mathrm{~d} x^{i} \wedge \mathrm{d} x^{j}-\mathrm{d} H \wedge \mathrm{d} t, \quad a_{i}=i_{\partial / \partial x^{i}} \omega
$$

where $i$ is the interior product operator for ordinary differential forms.

3. Some of the results in $\S 3$ can be seen in the light of the discussion in the previous subsection: By (3.24), $X\left(u^{\mu}\right)=P^{\mu}$ is the defining relation for the components of an arbitrary vector field $X$. The approach in this paper leads to concrete results in particular cases, once $X$ is chosen appropriately in each case, say $\left\langle\mathrm{d} u^{\mu}, X\right\rangle=: P^{\mu}=\tilde{R}^{\mu}$, as it was done for instance in $\S 4$. This fact can be recast in a more suggestive form, namely

$$
\left\langle\tilde{\alpha}^{\mu}, X\right\rangle=0, \quad \text { where } \quad \tilde{\alpha}^{\mu}:=\mathrm{d} u^{\mu}-\tilde{R}^{\mu} \rho
$$

that is, the $(N+1) 1$-forms $\tilde{\alpha}^{\mu}$ vanish along $X$. Notice that, owing to $\sum_{\mu} P^{\mu}=1$, we must

impose the condition $\sum_{\mu} \tilde{R}^{\mu}=1$, hence $\sum_{\mu} \tilde{\alpha}^{\mu}=0$, that is, the $\tilde{\alpha}^{\mu}$ are linearly dependent. Moreover, by (3.32), the correlation matrix of the lattice coordinates is $P^{\mu \nu}=\left\langle\tilde{\alpha}^{\mu} \bullet \tilde{\alpha}^{\nu}, X\right\rangle$ in close analogy to (5.24). In fact, (A.6ii) is just (5.13) written in the lattice coordinates $\left(u^{\mu}\right)$. To see this, we transform (A.6) to $\left(t, x^{i}\right)$ coordinates. Since $P^{\mu}=\tilde{R}^{\mu}$, the $\tilde{\alpha}^{\mu}$ become

$$
\begin{aligned}
\alpha^{0} & =\sum_{\mu}(-b) A_{\mu}^{0} \tilde{\alpha}^{\mu}=-b \sum_{\mu} \tilde{\alpha}^{\mu}=0 \\
\alpha^{i} & =\sum_{\mu} a_{i} A_{\mu}^{i} \tilde{\alpha}^{\mu}=\sum_{\mu} a_{i} A_{\mu}^{i} \mathrm{~d} u^{\mu}-\sum_{\mu} a_{i} A_{\mu}^{i} \tilde{R}^{\mu} \rho=\mathrm{d} x^{i}+\frac{a_{i}}{b}(A \tilde{R})^{i} \mathrm{~d} t
\end{aligned}
$$

By setting $\left(a_{i} / b\right)(A \tilde{R})^{i}=R^{i}$, the second equation becomes (5.13), hence (A.6i) is transformed to (5.14).

\section{APPENDIX B}

As mentioned in $\S 5$ remark (5), in specific cases, instead of expanding the evolution equation (5.10) as in (5.12), it is possible to write it explicitly in terms of appropriate difference operators which in the continuous limit gives the corresponding form of (5.20). As an illustration, we present here an $N$-dimensional example which generalizes that of $\S 2$. We employ the definitions and notations of $\S \S 3,5$.

The transformation (5.1) is chosen as follows $(i=1,2, \ldots, N)$

$$
t=-b \sum_{\mu} u^{\mu}, \quad x^{i}=a_{i}\left(u^{0}+\cdots+u^{i-1}-u^{i}+u^{i+1}+\cdots+u^{N}\right) .
$$

so that its inverse (5.4) and the commutation relations (5.8) become

$$
u^{0}=\frac{n-2}{2} \frac{t}{b}+\frac{1}{2} \sum_{i=1}^{N} \frac{x^{i}}{a_{i}}, \quad u^{i}=-\frac{1}{2}\left(\frac{t}{b}+\frac{x^{i}}{a_{i}}\right)
$$




$$
\begin{gathered}
\mathrm{d} t \bullet \mathrm{d} t=-b \mathrm{~d} t, \quad \mathrm{~d} t \bullet \mathrm{d} x^{i}=-b \mathrm{~d} x^{i}, \quad \mathrm{~d} x^{i} \bullet \mathrm{d} x^{i}=-\frac{a_{i}^{2}}{b} \mathrm{~d} t, \\
\mathrm{~d} x^{i} \bullet \mathrm{d} x^{j}=-\frac{a_{i} a_{j}}{b} \mathrm{~d} t+a_{j} \mathrm{~d} x^{i}+a_{i} \mathrm{~d} x^{j}
\end{gathered}
$$

In the limit (5.3), eq(5.21) takes the form

$$
\mathrm{d} t \bullet \mathrm{d} t=0, \quad \mathrm{~d} t \bullet \mathrm{d} x^{i}=0, \quad \mathrm{~d} x^{i} \bullet \mathrm{d} x^{j}=-h_{i j} \mathrm{~d} t,
$$

For the differential of a function of $u^{\mu}$

$$
\mathrm{d} f=\sum_{\mu}\left[f\left(u^{0}, \ldots, u^{\mu-1}, u^{\mu}+1, u^{\mu+1}, \ldots, u^{n}\right)-f\left(u^{0}, \ldots, u^{n}\right)\right] \mathrm{d} u^{\mu} .
$$

As a consequence eq(5.5) takes the form

$$
\begin{aligned}
\mathrm{d} f= & \frac{1}{2 b}\left[-\sum_{i=1}^{N} f\left(t-b, x+a, x^{i}-a_{i}, x+a\right)+(n-2) f(t+b, x+a)+2 f(t, x)\right] \mathrm{d} t \\
& +\sum_{i}\left(\bar{\partial}_{i} f\right)\left(t-b, x+a, x^{i}, x+a\right) \mathrm{d} x^{i},
\end{aligned}
$$

with

$$
\left(\bar{\partial}_{i} f\right)\left(\ldots, x^{i}, \ldots\right):=\frac{1}{2 a_{i}}\left[f\left(\ldots, x^{i}+a_{i}, \ldots\right)-f\left(\ldots, x^{i}-a_{i}, \ldots\right)\right]
$$

After a lengthy calculation we obtain

$$
\begin{aligned}
\mathrm{d} f= & {\left[\left(\partial_{-t} f\right)(t, x)-\sum_{i=1}^{N} \frac{a_{i}^{2}}{2 b}\left(\Delta_{i} f\right)\left(t-b, x+a, x^{i}, x+a\right)\right.} \\
& \left.+\sum_{1 \leq i<j \leq N} \frac{a_{i} a_{j}}{b}\left(\partial_{+i} \partial_{+j} f\right)\left(t-b, x+a, x^{i}, x, x^{j}, x+a\right)\right] \mathrm{d} t \\
& +\sum_{i}\left(\bar{\partial}_{i} f\right)\left(t-b, x+a, x^{i}, x+a\right) \mathrm{d} x^{i},
\end{aligned}
$$

where

$$
\begin{gathered}
\left(\partial_{-t} f\right)(t, x):=\frac{1}{b}[f(t, x)-f(t-b, x)] \\
\left(\Delta_{i} f\right)\left(\ldots, x^{i}, \ldots\right):=\frac{1}{a_{i}^{2}}\left[f\left(\ldots, x^{i}+a_{i}, \ldots\right)+f\left(\ldots, x^{i}-a_{i}, \ldots\right)-2 f\left(\ldots, x^{i}, \ldots\right)\right] .
\end{gathered}
$$

(cf. (2.7)-(2.10)). Evidently, in the limit (5.3), eq(B.6) gives

$$
\begin{aligned}
& \mathrm{d} f=\left[\left(\partial_{t} f\right)(t, x)-\right. \sum_{i=1}^{N} h_{i i}\left(\partial_{i}^{2} f\right)(t, x)+ \\
&\left.\sum_{1 \leq i<j \leq N} h_{i j}\left(\partial_{i} \partial_{j} f\right)(t, x)\right] \mathrm{d} t+\sum_{i}\left(\partial_{i} f\right)(t, x) \mathrm{d} x^{i}
\end{aligned}
$$


By (3.24), a vector field in this calculus has the form

$$
(X f)(u)=\sum_{\mu=0}^{N} P^{\mu}\left(\partial_{+\mu} f\right)(u)=\sum_{\mu=0}^{N} P^{\mu}\left(f\left(u, u^{\mu}+1, u\right)-f(u)\right),
$$

or, by (B.1)

$$
\begin{aligned}
(X f)(t, x)= & X^{t}(t, x)\left[\left(\partial_{-t} f\right)(t, x)-\sum_{i=1}^{N} \frac{a_{i}^{2}}{2 b}\left(\Delta_{i} f\right)\left(t-b, x+a, x^{i}, x+a\right)\right. \\
& \left.+\sum_{1 \leq i<j \leq N} \frac{a_{i} a_{j}}{b}\left(\partial_{+i} \partial_{+j} f\right)\left(t-b, x+a, x^{i}, x, x^{j}, x+a\right)\right] \\
& +\sum_{i} X^{i}(t, x)\left(\bar{\partial}_{i} f\right)\left(t-b, x+a, x^{i}, x+a\right), \\
X^{t}=-b\left(P^{0}+\cdots+\right. & \left.P^{n}\right), \quad X^{i}=a_{i}\left(P^{0}+\cdots+P^{i-1}-P^{i}+P^{i+1}+\cdots+P^{n}\right)
\end{aligned}
$$

Then by (3.17), $X$ defines a flow on $\mathcal{M}$, if and only if

$$
P^{\mu} P^{\nu}=\delta^{\mu \nu} P^{\mu}
$$

and has $N+1$ solutions with $P^{\mu}=1, P^{\nu}=0$, if $\nu \neq \mu$, for $\mu=0,1, \ldots, N$. Using (B.9) we readily get that in $\left(t, x^{i}\right)$ coordinates, the evolution generator for observables, eq( $[3.34)$, has components $\left(1, \pm a_{i} / b\right)$, thus obtaining the $N$-dimensional generalization of the "randomwalk model" of $\S 2$ (see eq(2.17)).

Acknowledgement: The authors would like to thank F. Müller-Hoissen for his critical remarks on the original draft of the paper. C.T. was partially supported by the University of the Aegean, under grant EPEAEK/397 and the paper was completed while he was at the University of the Aegean, on leave from the University of Crete. He would also like to thank all the members of the Department of Mathematics of the University of the Aegean for their hospitality.

\section{References}

[1] Bratelli O. and Robinson D. W., 1987, Operator Algebras and Quantum Statistical Mechanics 1, (New York: Springer), ch.2.3.5.

[2] Connes A. and Lott J., 1991, "Particle models and Noncommutative Geometry", Nucl. Phys. B (Proc. Suppl.), 18, 29-47.

[3] Connes A., 1994, Nonommutative Geometry, (San Diego: Academic Press).

[4] Madore J., 1995, An introduction to Noncommutative Differential Geometry and its applications, (Cambridge: Cambridge University Press). 
[5] Landi G., 1997, An introduction to noncommutative spaces and their geometries, (Berlin: Springer), hep-th/9701078.

[6] Varilly J.C., 1997, "An introduction to Noncommutative Geometry", Lectures at EMS Summer School on Noncommutative Geometry and Applications, Sept 1997, physics/9709045.

[7] Dimakis A. and Müller-Hoissen F., 1992, "Noncommutative Differential Geometry, Gauge Theory and Gravitation", preprint GOET-TP 33/92.

[8] Dimakis A. and Müller-Hoissen F., 1992 "Quantum Mechanics as non-commutative symplectic geometry", J.Phys A: Math.Gen. 25 5625-5648.

[9] Dimakis A. and Müller-Hoissen F., 1993 "Stochastic Differential Calculus, the Moyal *-product and non-commutative geometry", Lett. Math. Phys. 28 123-137.

[10] Dimakis A. and Müller-Hoissen F., 1993 "Non-Commutative Differential Calculus and Lattice Gauge Theory", J.Phys A: Math.Gen. 26 1927-1949.

[11] Dimakis A. and Müller-Hoissen F., 1994 "Discrete Differential Calculus, Graphs, Topologies and Gauge Theory", J.Math.Phys 35 6703-6735.

[12] Dimakis A., Müller-Hoissen F. and Vanderseypen F, 1995 "Discrete Differential Manifolds and Dynamics on Networks", J.Math.Phys 36 3771-3791.

[13] Dimakis A. and Tzanakis C., 1996 "Non-commutative geometry and the kinetic theory of open systems", J.Phys A: Math.Gen. 29 577-594.

[14] Dimakis A. and Müller-Hoissen F., 1996 "Soliton equations and the zero curvature condition in non-commutative geometry", J.Phys A: Math.Gen. 29 7279-7286.

[15] Dimakis A. and Tzanakis C., 1997 "Non-commutative geometry and its relation to Stochastic Calculus and Symplectic Mechanics", Proc. of the 4th Intern. Congress in Geometry, ed N.K. Artemiadis and N.K. Stefanidis, (Thessaloniki: Academy of Athens and University of Thessaloniki), 137-146.

[16] Dimakis A. and Müller-Hoissen F., 1997 "Non-commutative geometry and integrable models", Lett. Math. Phys. 39 69-79.

[17] Dimakis A. and Müller-Hoissen F., 1999 "Discrete Riemannian Geometry", J.Math.Phys 40(3), 1518-1548.

[18] Müller-Hoissen F., 1997 "Introduction to noncommutative geometry of commutative algebras and applications in physics" in Recent developments in Gravitation and Mathematical Physics:, Proc. of the 2nd Mexican School on Gravitation and Mathematical Physics, eds. A. Garcia, C. Lämmerzahl, A. Macias, T. Matos, and D. Nunez, Konstanz: Science Network Publishing 1998, http://kaluza.physik.uni-konstanz.de/2MS 
[19] Dimakis A. and Müller-Hoissen F., 1997, "Some aspects of noncommutative geometry and physics" in Contribution to the 21st Johns Hopkins Workshop, Lanzhou, China 1997, physics/9712004.

[20] Baehr H.C., Dimakis A. and Müller-Hoissen F., 1995 "Differential Calculi on Commutative Algebras", J.Phys A: Math.Gen. 28 3197-3222.

[21] Emery M., 1989, Stochastic Calculus on Manifolds, Berlin: Springer.

[22] Dimakis A. and Müller-Hoissen F., 1993, "Noncommutative differential calculus: quantum groups, stochastic processes and the antibracket", Proc. of the XXII Intern. Conference on Differential Geometric Methods in Theoretical Physics, Ixtapa, Mexico, published in Adv. Applied Clifford Algebras (Proc. Suppl.), 4(S1), 113-124.

[23] Lazota A. and Mackey M.C., 1985, Probabilistic properties of deterministic systems, London: Cambridge University Press.

[24] Chandrasekhar S., 1943, "Stochastic problems in physics and astronomy", Rev. Mod. Phys. 15(1), 1-89, reprinted in Selected papers on Noise and Stochastic Processes, ed. N. Wax, New York: Dover, 1956.

[25] Résibois P. and de Leener M., 1978, Classical Kinetic Theory of Fluids, (New York: Wiley).

[26] Balescu R., 1975, Equilibrium and Non-equilibrium Statistical Mechanics, New York: Wiley.

[27] Spohn H., 1980, "Kinetic equations from Hamiltonian dynamics: Markovian limits", Rev. Mod. Phys. 53, 569-615.

[28] Spohn H., 1991, Large-Scale Dynamics of interacting particles, Berlin: Springer 1991.

[29] Liboff R.L., 1969, Introduction to the theory of kinetic equations, Chichester: Wiley.

[30] Tzanakis C. and Grecos A.P., 1999, "Classical markovian kinetic equations:Explicit form and H-theorem", Transport Theory and Statistical Physics, 28 (4), 325-348.

[31] Kac M., 1956, "Random walk and the theory of Brownian motion", in Selected papers on Noise and Stochastic Processes, ed. N. Wax, New York: Dover, 1956.

[32] Roepstorff G., 1994, Path Integral approach to Quantum Physics, (Berlin: Springer).

[33] Bombelli L., Lee J., Meyer D. and Sorkin R.D., 1987, "Space-time as a causal set", Phys. Rev. Lett. 59, 521.

[34] Sorkin R.D., 1991, "Finitary substitute for continuous topology", Int. J. Theor. Phys., 30, 923-948.

[35] Loll R., 1998, "Discrete approaches to Quantum Gravity in four dimensions", http: www.livingreviews.org/Articles/Volume1/1998-13loll. 
[36] Gibbs P., 1996, "The small-scale structure of space-time: A bibliographical review", hep-th/950617.

[37] Ross S., 1994, A first course in probability, 4th edition, Englewood Cliffs: Prentice-Hall.

[38] Grecos A.P. and Tzanakis C., 1988, "Kinetic theory in the weak-coupling approximation: I. Formal theory and application to classical open systems", Physica A151, 61-89.

[39] Gardiner C.W., 1985, Handbook of Stochastic Methods, (Berlin: Springer).

[40] van Kampen N.G., 1981, Stochastic Processes in Physics and Chemistry, (Amsterdam: North Holland).

[41] Mazo R., 1978, "Aspects of the theory of Brownian motion, in Stochastic Processes in Nonequilibrium Systems, (eds) L. Garido, P. Seglar, P.J. Shepherd, Lecture Notes in Physics, 84, (Berlin: Springer), pp.53-81.

[42] Blanchad Ph., Combe Ph. and Zheng W., 1987, Mathematical and physical aspects of Stochastic Mechanics, Lecture Notes in Physics, 281, (Berlin: Springer)

[43] Tzanakis C. and Grecos A.P., 1988, "Kinetic theory for a classical test-particle model in the weak-coupling approximation", Physica A149, 232-244.

[44] Tzanakis C., 1988, "Kinetic theory in the weak-coupling approximation: II. A classical harmonic oscillator model", Physica A151, 90-112.

[45] Frigerio A. and Gorini V., 1984, "Diffusion processes, Quantum Dynamical Semigroups and the classical KMS condition" J. Math. Phys., 25, 1050-1065

[46] Tzanakis C., Grecos A.P. and Hatjimanolaki P., 1998, "Generalized Moyal structures in phase space, master equations and their classical limit: II. Applications to harmonic oscillator models", Physica A256, 112-128.

[47] Arnold L., 1973, Stochastic Differential Equations, (New York: Wiley).

[48] Nelson E., 1967, Dynamical Theories of Brownian Motion, (Princeton: Princeton University Press).

[49] Nelson E., 1985, Quantum Fluctuations, (Princeton: Princeton University Press). 